\title{
PRMT5, regulated by IncRNA ZFAS1/miR-150-5p, promoted androgen-independent prostate cancer migration and invasion
}

\author{
Hao Shen ${ }^{1}$, Lei Wang ${ }^{1}$, Ling Zhang ${ }^{2}$, Xiao-Dong Weng ${ }^{1}$, Du Yang ${ }^{1}$, Xiu-Heng Liu ${ }^{1}$
}

\begin{abstract}
'Department of Urology, Renmin Hospital of Wuhan University, Wuhan, Hubei, China 2Department of Pathology, Jingzhou Central Hospital, The Second Clinical Medical College, Yangtze University, Jingzhou, Hubei, China
\end{abstract}

Submitted: 19 September 2019

Accepted: 5 April 2020

Arch Med Sci

DOI: https://doi.org/10.5114/aoms.2020.97987

Copyright @ 2020 Termedia \& Banach

\begin{abstract}
Introduction: Prostate cancer ( $\mathrm{PCa}$ ) is the most common male genitourinary malignancy in the world. The protein arginine methyltransferase 5 (PRMT5) is one of the main members of the type II PRMT family, which was reported to regulate androgen-dependent PCa cell proliferation. However, the upstream regulators of PRMT5 and its effects on androgen-independent PCa metastasis remained unclear. In the present study, we investigated whether PRMT5 could be a novel diagnostic marker and be used as a therapeutic target in PCa, to explore the possible molecular mechanism, and to understand the clinical importance of PRMT5 in PCa.

Material and methods: The present study evaluated PRMT5 expression levels in PCa and normal prostate samples using public datasets, including TCGA, GEPIA and GSE21032. Furthermore, CCK-8 assay, flow cytometer assay, and transwell assay were conducted to detect the roles of PRMT5. Luciferase reporter assay was used to determine the relationship among ZFAS1/miR-150-5p/PRMT5.

Results: Our results showed that PRMT5 was overexpressed in PCa samples. PRMT5 significantly promoted androgen-dependent PCa proliferation and cell cycle progression and suppressed cell apoptosis. However, PRMT5 did not affect androgen-independent PCa proliferation but it could significantly induce androgen-independent PCa metastasis. Knockdown of PRMT5 suppressed, whereas overexpression of PRMT5 induced, cell migration and invasion in androgen-independent DU145 and PC-3 cells. Moreover, our results showed that the ZFAS1/miR-150-5p axis regulated PRMT5 expression in PCa cells. Furthermore, the study showed that ZFAS1 and PRMT5 were overexpressed and miR-150-5p was down-regulated in PCa samples. Higher expression levels of ZFAS1 and PRMT5 were correlated with shorter disease-free survival time in PCa patients.

Conclusions: These results showed that PRMT5 may be a therapeutic target for PCa.
\end{abstract}

Key words: cell proliferation, metastasis, therapeutic target.

\section{Introduction}

Emerging studies have demonstrated that the changes of protein post-translational modification in oncogenes (such as VEGFR1 and MAP3K2) and tumor suppressors (such as p53 and RB1) [1-5] play an

\author{
Corresponding author: \\ PhD Xiu-Heng Liu \\ Department of Urology \\ Renmin Hospital \\ of Wuhan University \\ Wuhan, Hubei 430060, China \\ E-mail: Drliuxh@hotmail.com
}


important role in human tumorigenesis. The protein arginine methyltransferase (PRMT) family plays important roles in regulating the post-translational modification of multiple proteins, including histone proteins and non-histone proteins [6]. Emerging studies have indicated that PRMT family members were dysregulated and involved in regulating the progression of human cancers, such as breast cancer, and lung cancer. Among these PRMT family members, the important roles of PRMT1, PRMT2, PRMT5 and PRMT7 have been demonstrated in cancers [7].

Prostate cancer ( $\mathrm{PCa}$ ) is the most common male genitourinary malignancy in the world [8]. However, the biological mechanisms regulating PCa progression have remained elusive. Androgen receptor (AR) is the most important regulator involved in modulating $\mathrm{PCa}$ proliferation, invasion and glycolysis $[9,10]$. Speckle-type POZ (SPOP) is one of the most frequently mutated genes in $\mathrm{PCa}$, whose mutations were associated with drug resistance, lipid accumulation and genomic stability [11-13]. Recent studies showed PRMT family members regulated the tumorigenesis, progression and development of PCa through affecting multiple signaling pathways [14, 15]. For example, PRMT6 played an oncogenic role of in PCa [16]. PRMT10 is required for androgen-dependent proliferation of $P C a$ LNCaP cells [17]. Therefore, to establish effective indicators of prognosis and therapeutic strategies against this disease, we need a clearer realization of the molecular pathology of PCa.

PRMT5 is a main member of the type II PRMT family [18]. In human cells, PRMT5 commonly interacts with MEP50 at the catalytic site and produces monomethylarginine [19]. Recent studies have indicated that PRMT5 plays a regulatory role in promoting cancer progression [20]. PRMT5 was observed to be overexpressed in multiple cancers, such as melanoma, lung cancer, ovarian cancer, breast cancer and prostate cancer [21]. More PRMT5 was expressed in PCa tissues, which is positively correlated with AR expression. PRMT5 promotes PCa cell growth by epigenetic activation of AR transcription [22]. Inhibition of PRMT5 down-regulates AR expression and inhibits the growth of multiple AR-positive PCa cells [23]. A previous study showed that ERG recruited PRMT5 to AR and methylate AR Arginine 761, which suppressed the recruitment and transcription of AR target genes [24]. However, the effects of PRMT5 on PCa metastasis and the up-stream regulators of PRMT5 in PCa remained to be further investigated.

In the present study, the expression of PRMT5 in PCa was analyzed by using public datasets and the molecular functions of PRMT5 in PCa were investigated using loss-of function assays. The aims of the study were to investigate whether PRMT5 could be a novel diagnostic marker and used as a therapeutic target in $\mathrm{PCa}$, to explore the possible molecular mechanism, and to understand the clinical importance of PRMT5 in prostate carcinogenesis.

\section{Material and methods}

\section{Cell culture}

LNCaP, 22RV1, DU145, and PC-3 were purchased from the American Type Culture Collection (Manassas, VA) and confirmed by short tandem repeat (STR) analysis or mycoplasma detection, DNA fingerprinting, isozyme detection, and cell vitality detection. These cells were cultured in RPMI 1640 medium (with 10\% fetal bovine serum, Biochrom) at $37^{\circ} \mathrm{C}$ and in a $5 \% \mathrm{CO}_{2}$ humidified incubator for further study.

\section{RNA interference}

The siRNAs against the PRMT5 (siPRMT5-1, siPRMT5-2) and negative control (siNC) were synthesized by GenePharma (Shanghai, China). The PCa cells were seeded and transfected in 12-well plates using Lipofectamine 3000 (Life Technologies), in accordance with the manufacturer's instructions. The following siRNAs were used: SiPRMT5-1: 5'- CAACAGAGAUCCUAUGAUU -3'; SIPRMT5-2: 5'- AAGAGGGAGUUCAUUCAGGAA -3'; and a scrambled siRNA control: 5'- AAGUGAUAGGAAGUCAGUACG -3'.

\section{Plasmids and cell transfection}

Human CDNA of PRMT5 was obtained from Jiahuai Han (Xiamen University) and cloned into plasmid pcDNA3.1(+) (Invitrogen). The PCa cells were seeded and transfected in 6-well plates with pcDNA3.1(+) or pcDNA3.1(+)-PRMT5 plasmid (800 ng/ well) using Lipofectamine 3000 (Life Technologies) according to the manufacturer's instructions.

\section{Real-time quantitative PCR}

Beyozol (Beyotime, China) was used to extract total RNA from PCa cells. BeyoRT First Strand cDNA Synthesis Kit (Beyotime, China) was used to perform the reverse transcription according to the manufacturer's instructions. Real-time quantitative PCR (RT-qPCR) was conducted using ABI Prism 7900HT (Applied Biosystems, Foster City, CA). Primers for PRMT5 were: forward, 5'- TGAATTGTCGCCTGAGTGC-3' and reverse, 5'- GGGATGCTCACACCATCAT - $3^{\prime}$. Primers for $\beta$-actin were: forward, 5'- GAGCTACGAGCTGCCTGACG -3' and reverse, 5'- CCTAGAAGCATTTGCGGTGG -3'. Primers were synthesized by GenePharma (Shanghai, China). $\beta$-actin was selected as a reference. The $2^{-\Delta \Delta c t}$ 
method was used to calculate the relative mRNA expression. Each sample was run in triplicate [25]

\section{Western blot analysis}

Cells were harvested $48 \mathrm{~h}$ post transfection, using RIPA lysis buffer RIPA (strong). BCA Protein Assay Kit (Beyotime, China) was used to detect the content of proteins according to the manufacturer's instructions. $12 \%(\mathrm{w} / \mathrm{v})$ polyacrylamide gel SDS-PAGE was used to separate proteins. $\beta$-actin was selected as a reference. PRMT5 and ACTB were detected by monoclonal antibodies for PRMT5 and ACTB (1: 1,000; Cell Signaling Technology, Beverly, MA, USA).

\section{Cell proliferation assay}

Cell Counting Kit-8 (CCK-8; Dojindo Laboratories, Kumamoto, Japan) was used to measure cell proliferation according to the manufacturer's instructions. After transfection, we measured the absorbance at $450 \mathrm{~nm}$ using a Microplate Reader at $0,24,48,72,96$, and $120 \mathrm{~h}$. The absorbance at $630 \mathrm{~nm}$ was selected as a reference. Each sample was run in triplicate.

\section{Cell cycle and cell apoptosis assay}

Cell Cycle and Apoptosis Analysis Kit (Beyotime, China) was used to perform the cell cycle assay according to the manufacturer's instructions. The cells were harvested $48 \mathrm{~h}$ post transfection. $1 \mathrm{ml}$ of pre-cool $70 \%$ ethanol was added and mixed by gently pipetting, then placed at $4^{\circ} \mathrm{C}$ for $12 \mathrm{~h}$. Then the cells were collected by centrifugation at $1000 \mathrm{~g}$, 3 min. The prepared Cell Cycle and Apoptosis Analysis Kit was added to each tube, and the cell pellet was slowly and fully resuspended, and left at $37^{\circ} \mathrm{C}$ for $30 \mathrm{~min}$ in the dark. The FACStar flow cytometer (Becton-Dickinson, San Jose, CA) was used to measure the cell cycle.

Annexin V-FITC Apoptosis Detection Kit (Beyotime, China) was used to perform the cell apoptosis assay according to the manufacturer's instructions. The cells were harvested and gently resuspended by adding $195 \mu$ l of Annexin V-FITC binding solution. Then $5 \mu$ l of Annexin V-FITC and $10 \mu \mathrm{l}$ of propidium iodide staining solution were added to the mixture and it was mixed gently. Then the mixture was incubated at room temperature for $15 \mathrm{~min}$ in the dark, then placed in an ice bath. A FACStar flow cytometer was used to measure the cell apoptosis.

\section{Cell migration and invasion assay}

Transwell plates $(8-\mu \mathrm{m}$ pore size, $6.5 \mathrm{~mm}$ diameter; Corning Life Sciences, Lowell, MA) with or without Matrigel (BD) were used to conduct cell migration and invasion assays according to the manufacturer's protocol. For migration, $100 \mu \mathrm{l}$ of medium containing $1.5 \times 10^{4}$ cells $(1 \%$ FBS) was added to the upper chamber. $700 \mu$ of medium (10\% FBS) was added to the lower chamber.

\section{ceRNA network construction}

The IncRNA-miRNA interactions and miRNA-mRNA interactions were predicted using StarBase V2.0 (http://starbase.sysu.edu.cn/starbase2/). The ceRNA regulatory network was established based on IncRNA-miRNA-mRNA axes by combining IncRNA-miRNA interactions with miRNA-target gene interactions. Cytoscape v3.6.0 was used to visualize the ceRNA network.

\section{Reporter constructs and luciferase assay}

The Dual-Luciferase Reporter Assay System (Promega, USA) was used to perform the Luciferase assay. The predicted binding sites for miR-150 in ZFAS1 and 3'-UTR of PRMT5 were inserted into psi-CHECK2 Dual-Luciferase miRNA Target Expression Vector (Promega, USA) within the Xhol/ Notl sites. Sequence integrity was confirmed by sequencing.

\section{Statistical analysis}

All data are shown as the mean \pm standard deviation (SD). The SPSS software package, version 15.0 (SPSS Inc., Chicago, IL) was used to perform statistical analysis. Student's t test was used to determine significant differences between two groups. In order to compare the significant differences among multiple groups (group $\geq 3$ ), oneway ANOVA followed by Tukey's test was used. Kaplan-Meier curves with log ranking tests were conducted to assess the correlation between PRMT5 or ZFAS1 and survival time in patients with PCa. A value of $p<0.05$ was selected as significant.

\section{Results}

\section{PRMT5 was overexpressed and correlated} with poor prognosis of $\mathrm{PCa}$

In order to further investigate the prognostic value of PRMT5 in PCa, we analyzed several public datasets, including TCGA and GEPIA. The results showed that the expression level of PRMT5 in PCa samples were significantly higher than that in normal prostate tissues by analyzing these datasets (Figures $1 \mathrm{~A}$ and B). Moreover, GSE21032 dataset analysis revealed that PRMT5 was up-regulated in metastasized PCa samples compared to primary PCa samples (GS < 7 PCa) and normal prostate tissues (Figure $1 \mathrm{C}$ ), suggesting that PRMT5 may be involved in regulating PCa metastasis. 
A

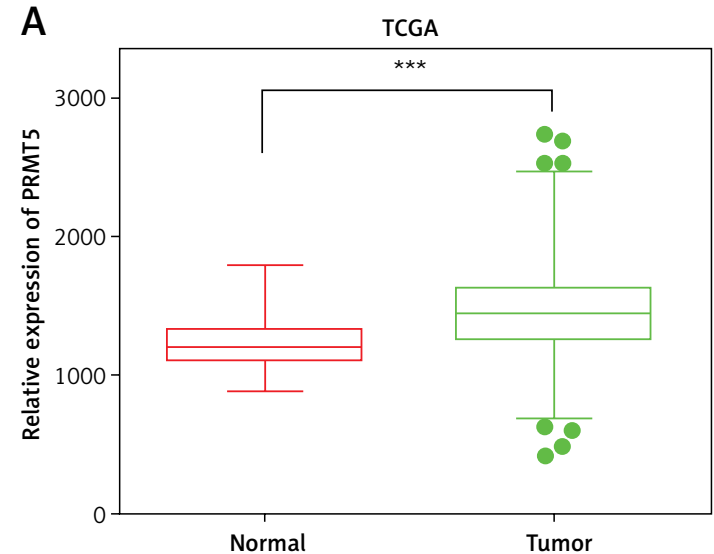

C

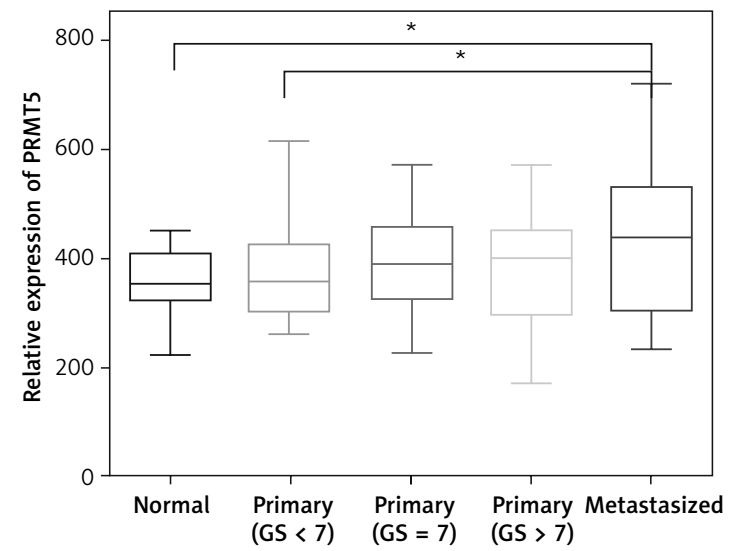

E

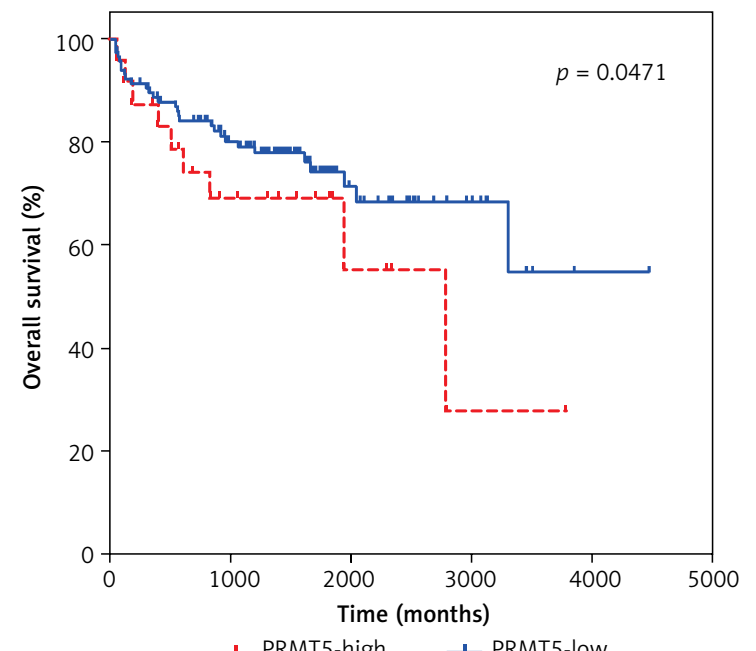

Furthermore, the association of PRMT5 expression with $B C R$-free survival (BFS) and overall survival (OS) in PCa patients was assessed by the Kaplan-Meier curve method. The cutoff point which divides all cases into PRMT5 high and PRMT5 low groups was the median PRMT5 mRNA expression in all PCa tissues. As shown in Figures $1 \mathrm{D}$ and $\mathrm{E}$, compared to PRMT5-high patients, the BFS $(p<0.01)$ and $\mathrm{OS}(p<0.05)$ rates were higher in PRMT5-low patients in both datasets $(p<0.05$, in
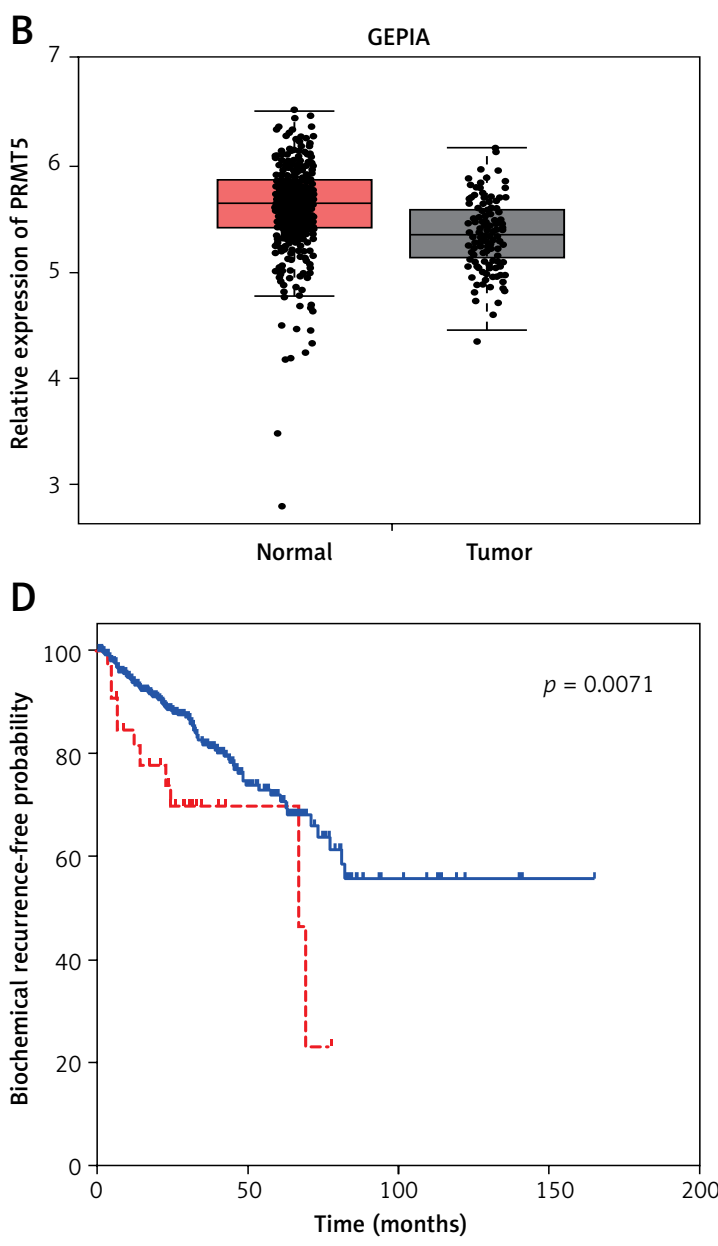

-L. PRMT5-high + PRMT5-low

Figure 1. PRMT5 was overexpressed and correlated with poor prognosis of PCa. A - TCGA and B GEPIA datasets revealed that the expression level of PRMT5 in PCa samples was significantly higher than that in normal prostate tissues. C - GSE21032 dataset analysis revealed that PRMT5 was up-regulated in metastasized PCa samples compared to primary PCa samples (GS $<7$ PCa) and normal prostate tissue. $\mathbf{D}$ - The BFS $(p<0.01)$ was higher in PRMT5-low patients compared to PRMT5-high patients. $\mathrm{E}-$ The OS $(p<0.05)$ rate was higher in PRMT5-low patients compared to PRMT5-high patients

both datasets), indicating that PRMT5 could be a potential biomarker for the prognosis of $\mathrm{PCa}$.

\section{Bioinformatics analysis of PRMT5 in PCa}

In order to explore the potential functions PRMT5 in PCa, the present study conducted co-expression analysis and protein-protein interaction (PPI) network analysis to identify the downstream targets of PRMT5. The top key up-regulated PPI (Figure $2 \mathrm{~A}$ ) and down-regulated PPI networks 
A

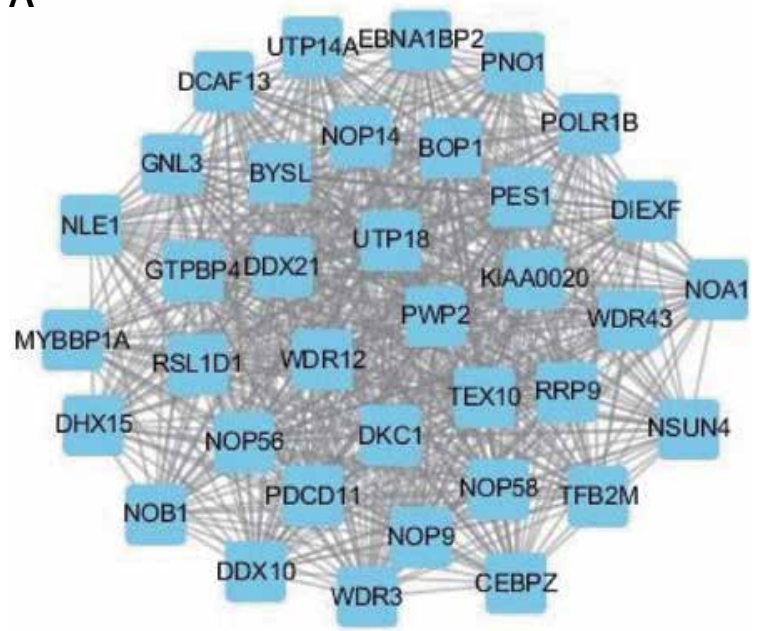

B

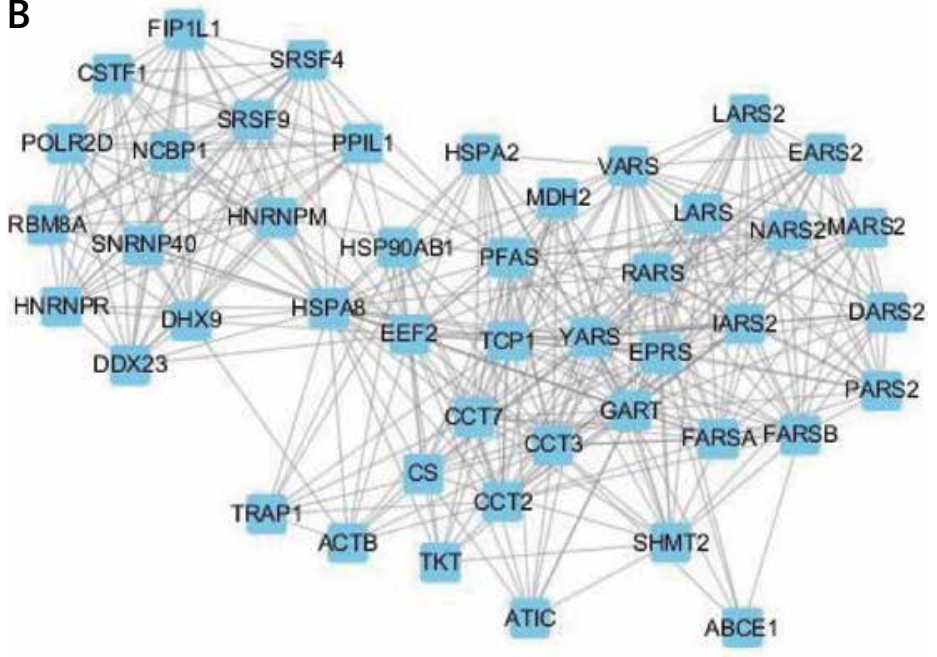

$\mathrm{C}$

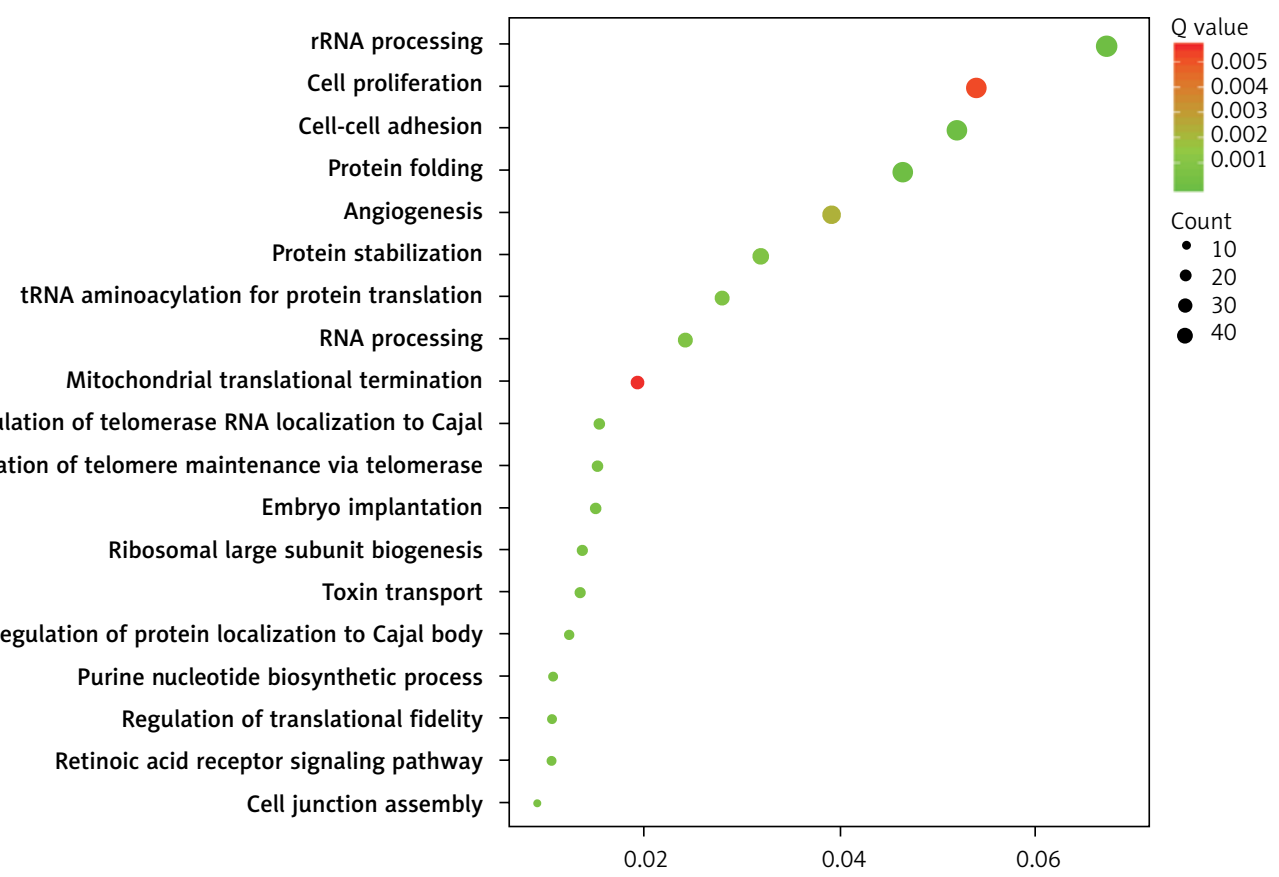

Figure 2. Bioinformatics analysis of PRMT5 in PCa. A - The top key up-regulated protein-protein interaction (PPI) networks of the downstream targets of PRMT5. B - The top key down-regulated PPI networks of the downstream targets of PRMT5. C - The biological processes and pathways involved in the top key up-regulated PPI networks 
D

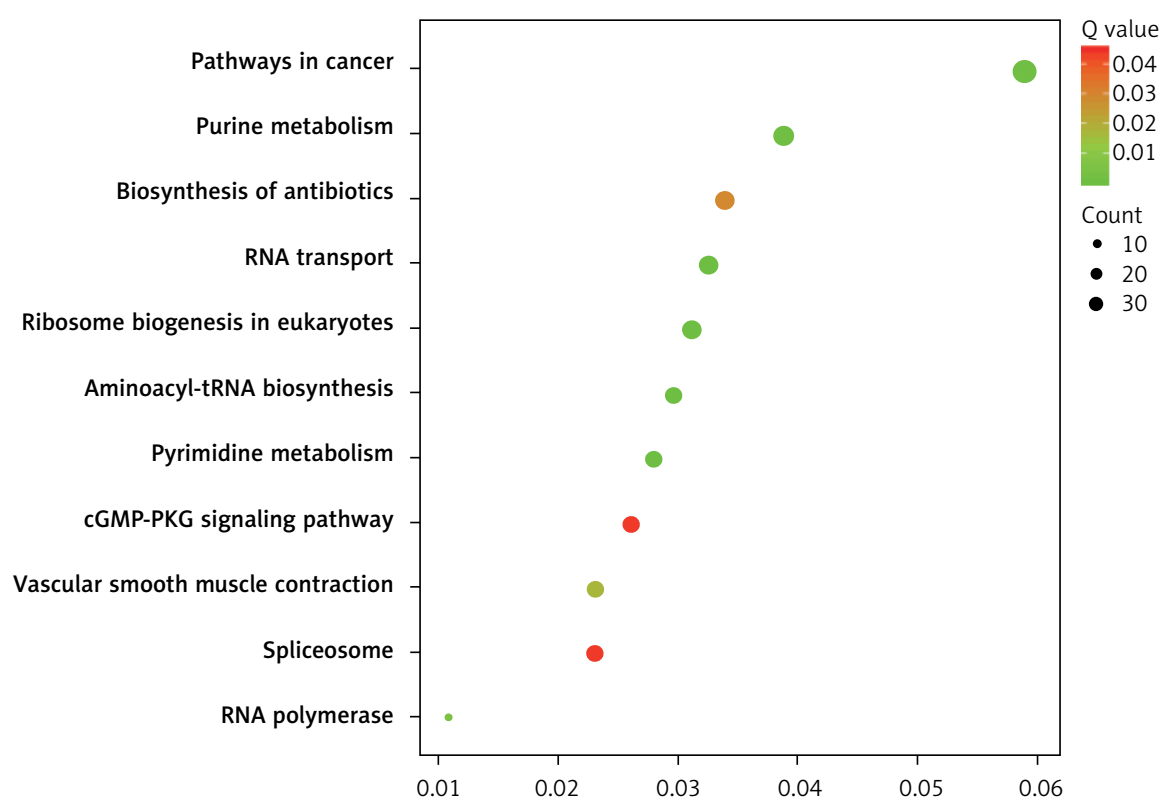

Figure 2. Cont. D - The biological processes and pathways involved in the top key down-regulated PPI networks

(Figure 2 C) are shown in Figure 2. Bioinformatics analysis revealed that PRMT5 was involved in regulating multiple biological processes and pathways, such as cell junction assembly, angiogenesis, cell-cell adhesion, cell proliferation, and spliceosome (Figures $2 \mathrm{~B}$ and D). These analyses were consistent with previous studies indicating that PRMT5 could promote PCa proliferation.

PRMT5 promotes proliferation of androgendependent PCa, not androgen-independent $\mathrm{PCa}$

The mRNA levels of PRMT5 were evaluated in the normal prostate WPMY-1 cells and human PCa cell lines, including LNCaP, DU145, and PC-3 by qRT-PCR analyses, respectively (Supplementary Figure S1 C). Our results showed that mRNA levels of PRMT5 were significantly induced in PCa LNCaP, 22RV1, DU145, and PC-3 compared to normal WPMY-1 cells. These results suggested that the overexpression of PRMT5 might be positively correlated with the progression of prostate cancer.

To characterize the function of PRMT5 in PCa, PRMT5 expression was knocked down at the mRNA and protein levels by PRMT5 siRNA. PRMT5-specific siRNA substantially decreased PRMT5 mRNA and protein levels in PCa cells compared with the siRNA control (NC) (Figures $3 \mathrm{~A}-\mathrm{C}$ ). Then, the present study detected the effect of PRMT5 on cell proliferation in PCa using the CCK8 assay. The results showed that the proliferation rate of the androgen-dependent PCa LNCaP cell line was significantly reduced after transfection with SiPRMT5-1 and SiPRMT5-2 compared to cells treated with the siNC $(p<0.001$, Figure 3 D). In- terestingly, our results showed that knockdown of PRMT5 did not affect the cell proliferation of androgen-independent PCa DU145 and PC-3 cells (Figures $3 \mathrm{E}$ and $\mathrm{F}$ ).

Subsequently, the present study validated the above finding through overexpressing PRMT5 in PCa cells. After the plasmid containing PRMT5 gene was transfected in $\mathrm{PCa}$, a significant increase of PRMT5 gene expression was detected (Figures $3 \mathrm{G}-1$ ). Our results showed that overexpression of PRMT5 remarkably enhanced the proliferation of LNCaP cells ( $p<0.05$, Figure $3 \mathrm{~J}$ ) and did not significantly promote DU145 and PC3 proliferation (Figure $3 \mathrm{~K}$ and L). Taken together, these results showed that PRMT5 promotes proliferation of androgen-dependent PCa, not androgen-independent PCa.

PRMT5 promotes the cell cycle progression of androgen-dependent PCa, not androgenindependent $\mathrm{PCa}$

Next, the effect of PRMT5 on the cell cycle progression of LNCaP and DU145 cells was assessed by flow cytometry. As presented in Figure 4, the results showed that knockdown of PRMT5 in LN$\mathrm{CaP}$ cells significantly increased the percentage of $\mathrm{G} 1$ phase cells and reduced the percentage of $S$ and G2/M phase cells compared to the NC group $(p<0.001$; Figure 4 A). However, our results showed that silencing of PRMT5 in DU145 cells did not change the percentage of G1 phase cells and $\mathrm{S}$ phage cells compared to the NC group (Figure $4 \mathrm{~B}$ ). Furthermore, the present study found that PRMT5 overexpression significantly promoted the cell cycle by reducing G1 phase and inducing $S$ phase cells in LNCaP cells, not in DU145 cells. 
A

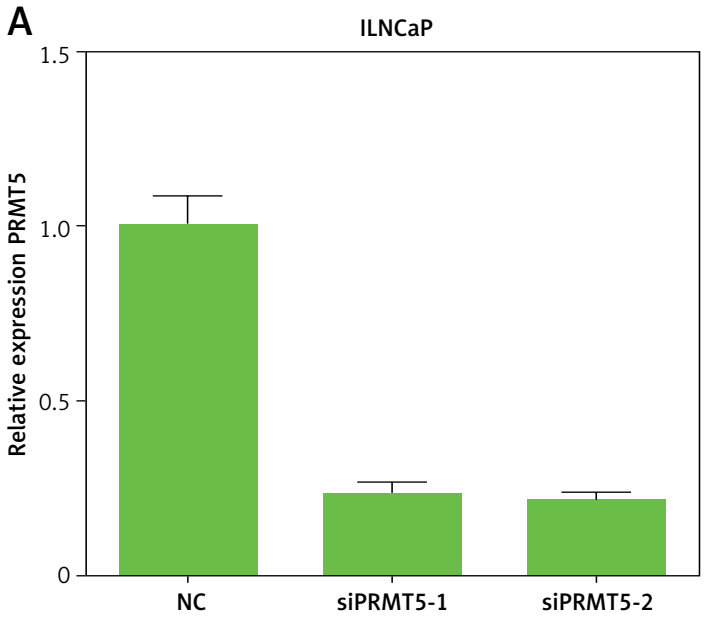

C

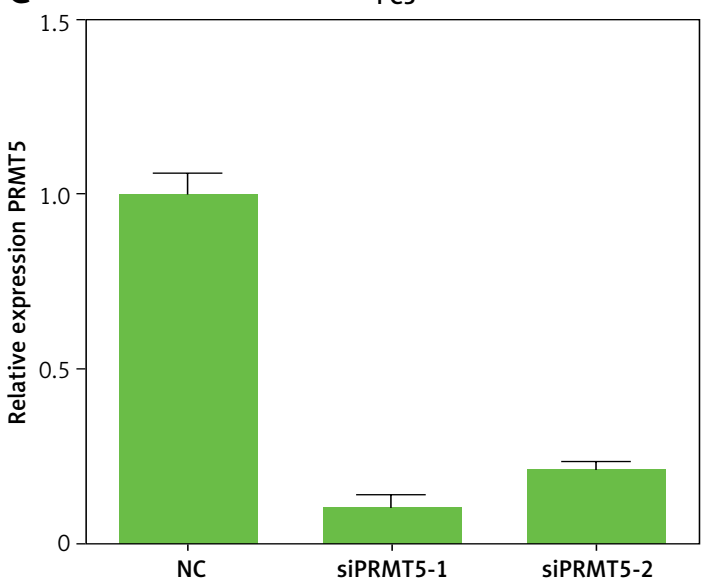

E

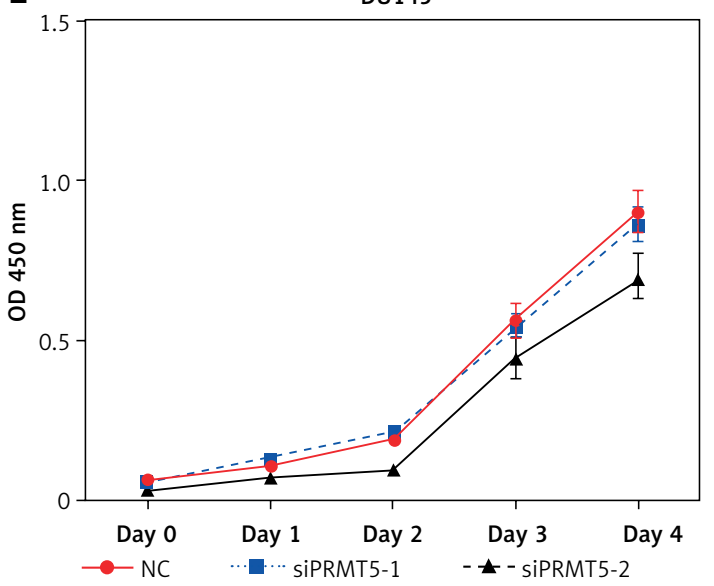

B

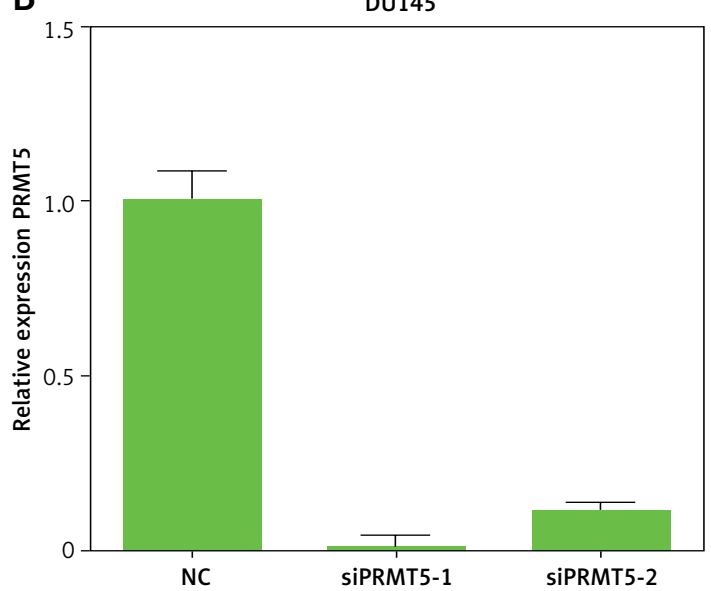

D

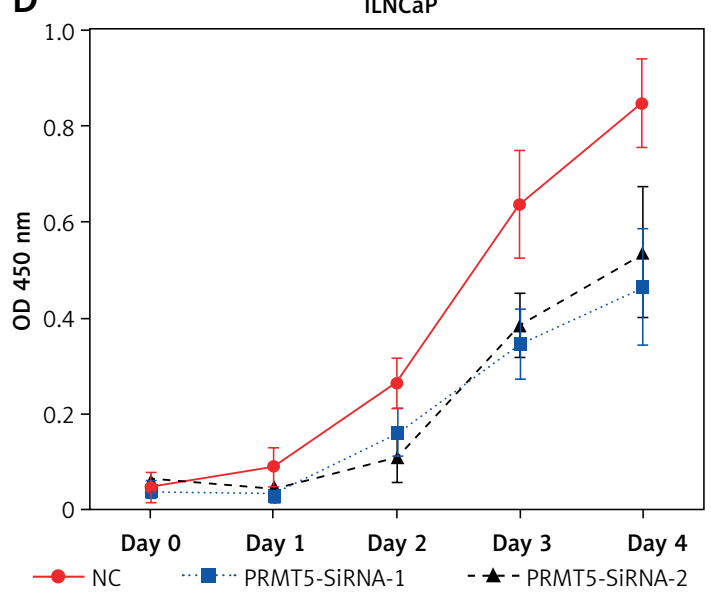

$\mathrm{F}$

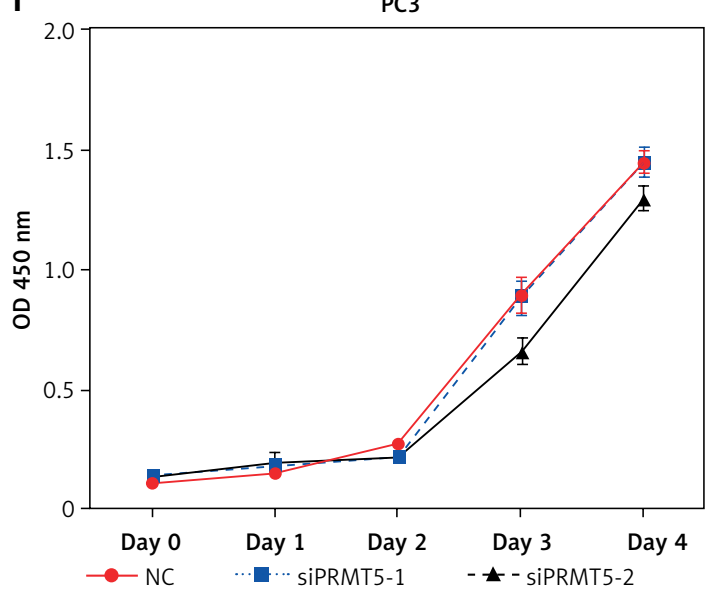

Figure 3. PRMT5 promotes proliferation of androgen-dependent PCa, not androgen-independent PCa. A-C - PRMT5-specific siRNA-1,2 substantially decreased PRMT5 mRNA levels in PCa cells LNCaP, DU145 and PC3. D - Knockdown of PRMT5 significantly reduced the proliferation rate of androgen-dependent PCa LNCaP cell line compared to cells treated with siNC $(p<0.001)$. E, F - Knockdown of PRMT5 did not affect the cell proliferation of androgen-independent PCa DU145 and PC-3 cells

PRMT5 suppressed cell apoptosis of androgen-dependent $\mathrm{PCa}$

Cell apoptosis plays crucial roles in regulating cancer proliferation. The present study conducted Annexin V-FITC/PI assay to detect the effect of PRMT5 on cell apoptosis in PCa. As shown in Figure 4 C, knockdown of PRMT5 using siPRMT5-1 and siPRMT5-2 significantly increased the early and late apoptotic cell fractions by $18 \%$ and $37.5 \%$ in LNCaP cells, respectively. However, knockdown 
G

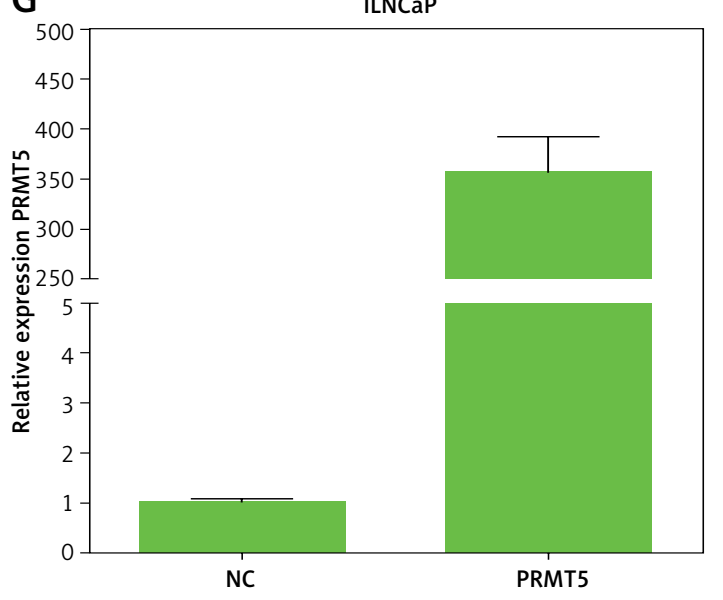

I

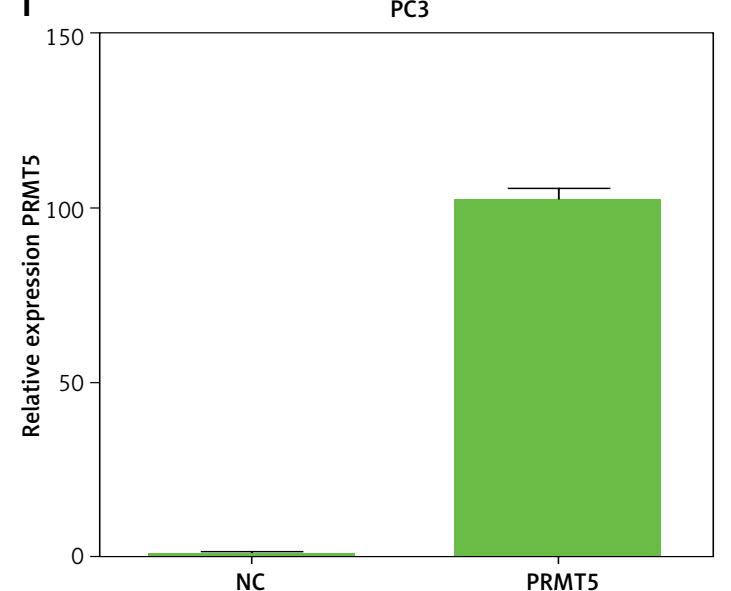

K

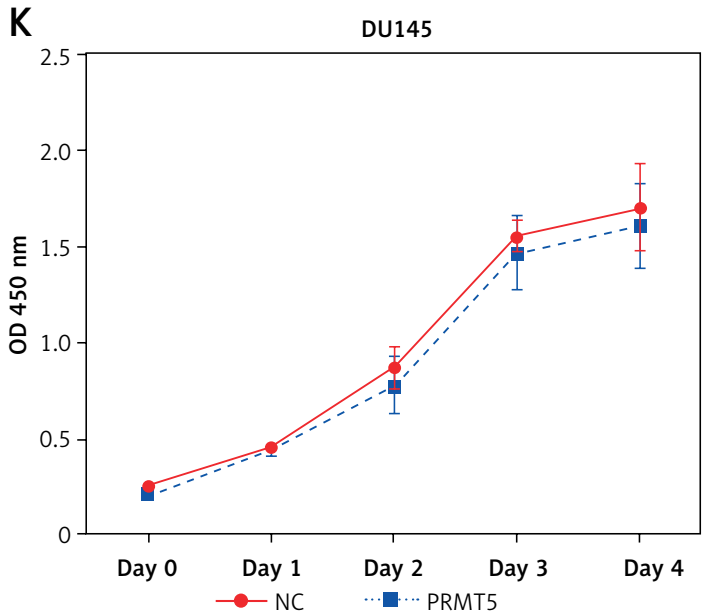

H

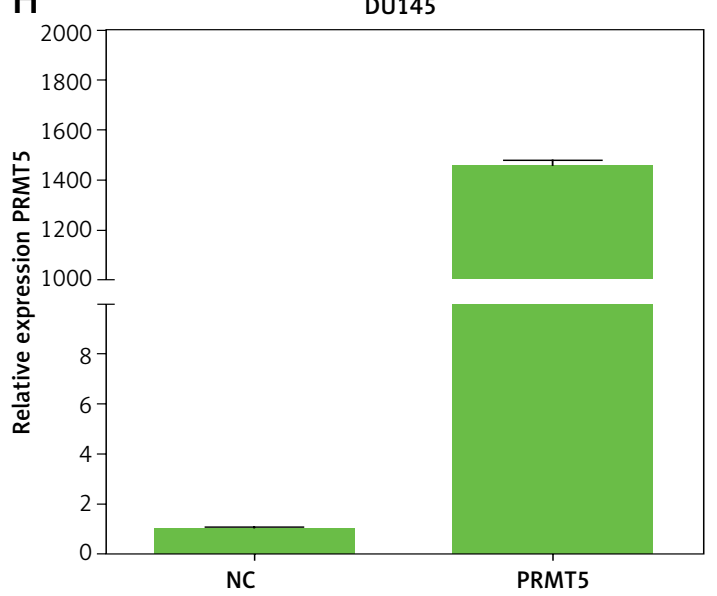

J

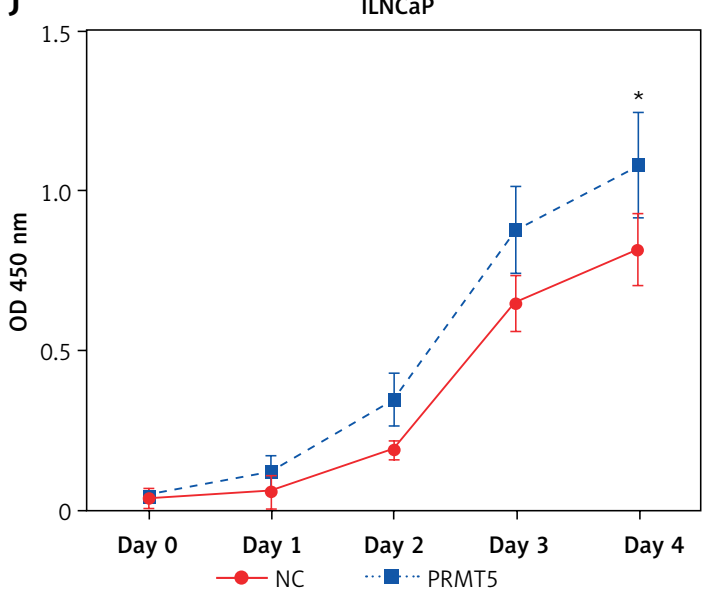

L

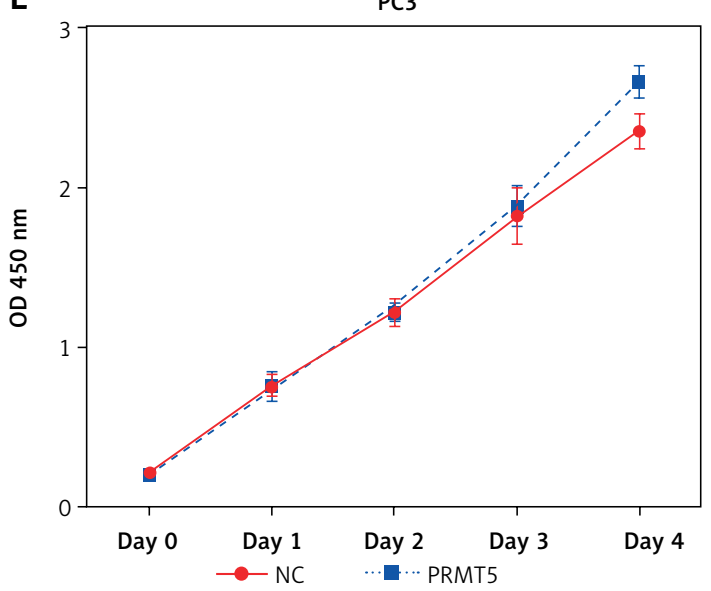

Figure 3. Cont. G-I - Overexpression of PRMT5 significantly increased PRMT5 gene expression in PCa cells LNCaP, DU145 and PC3. J - Overexpression of PRMT5 remarkably enhanced proliferation of LNCaP cells $(p<0.05)$. K, L - Overexpression of PRMT5 did not significantly promote DU145 and PC3 proliferation

of PRMT5 had no effect on the androgen-independent DU145 apoptosis (Figure 4 D).

PRMT5 promotes cell migration and invasion of androgen-independent PCa

Considering that PRMT5 did not affect the proliferation of androgen-independent $\mathrm{PCa}$, the present study hypothesized that PRMT5 might be involved in promoting androgen-independent PCa metastasis. First, the present study performed transwell assay to detect the effect of PRMT5 overexpression on PC-3 and DU145 cell migration. Our results showed that overexpression of PRMT5 remarkably enhanced PC-3 and DU145 

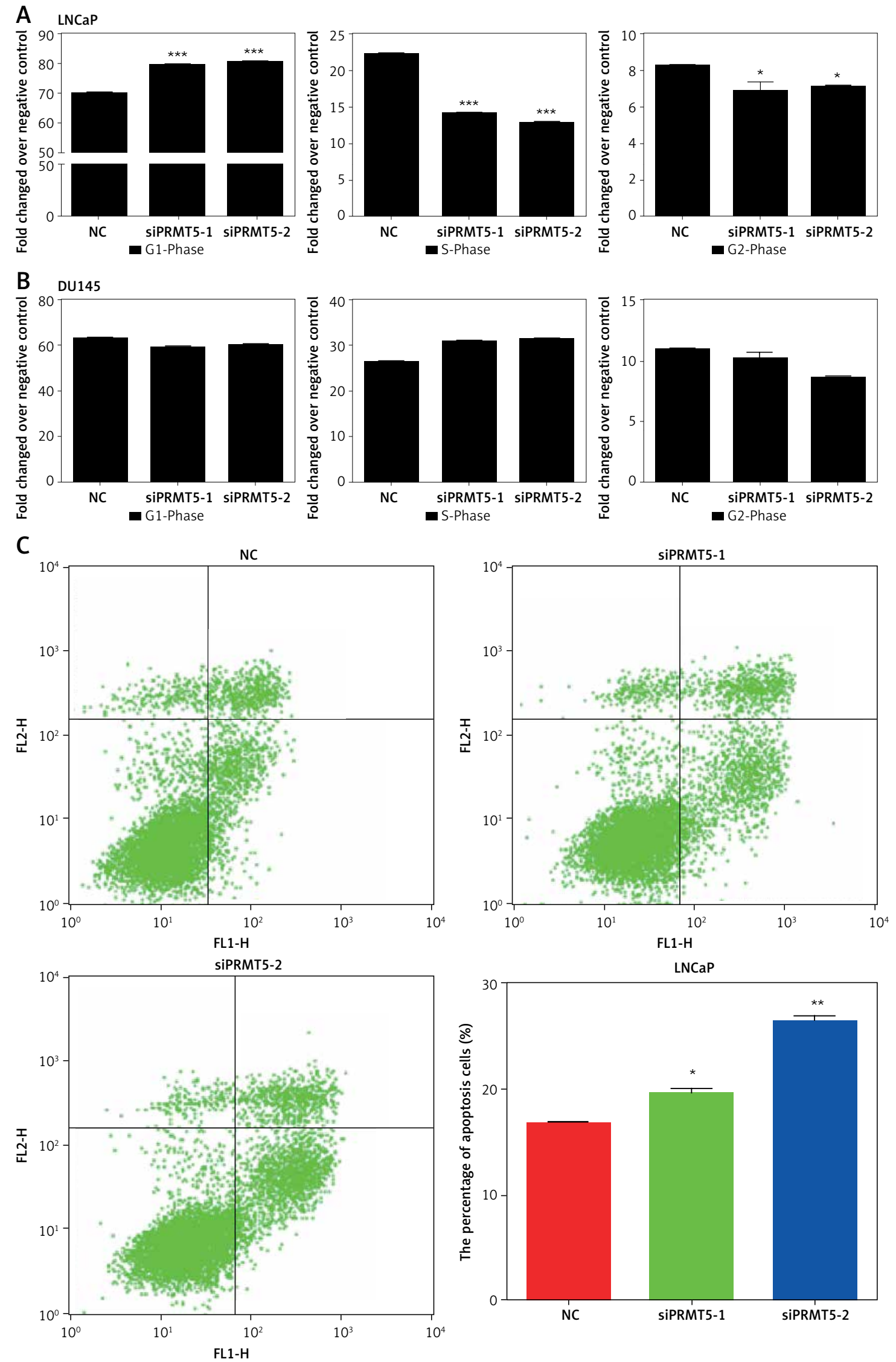

Figure 4. PRMT5 promotes cell cycle progression and cell apoptosis of androgen-dependent PCa, not androgen-independent PCa. A - Knockdown of PRMT5 in LNCaP cells significantly increased the percentage of G1 phase cells and reduced the percentage of $\mathrm{S}$ and $\mathrm{G} 2 / \mathrm{M}$ phase cells compared to NC group $(p<0.001)$. B - Knockdown of PRMT5 in DU145 cells did not change the percentage of G1 phase cells and S phage cells compared to NC group. C - Knockdown of PRMT5 significantly increased the early and late apoptotic cell fractions by $18 \%$ and $37.5 \%$ in $\mathrm{LNCaP}$ cells, respectively 

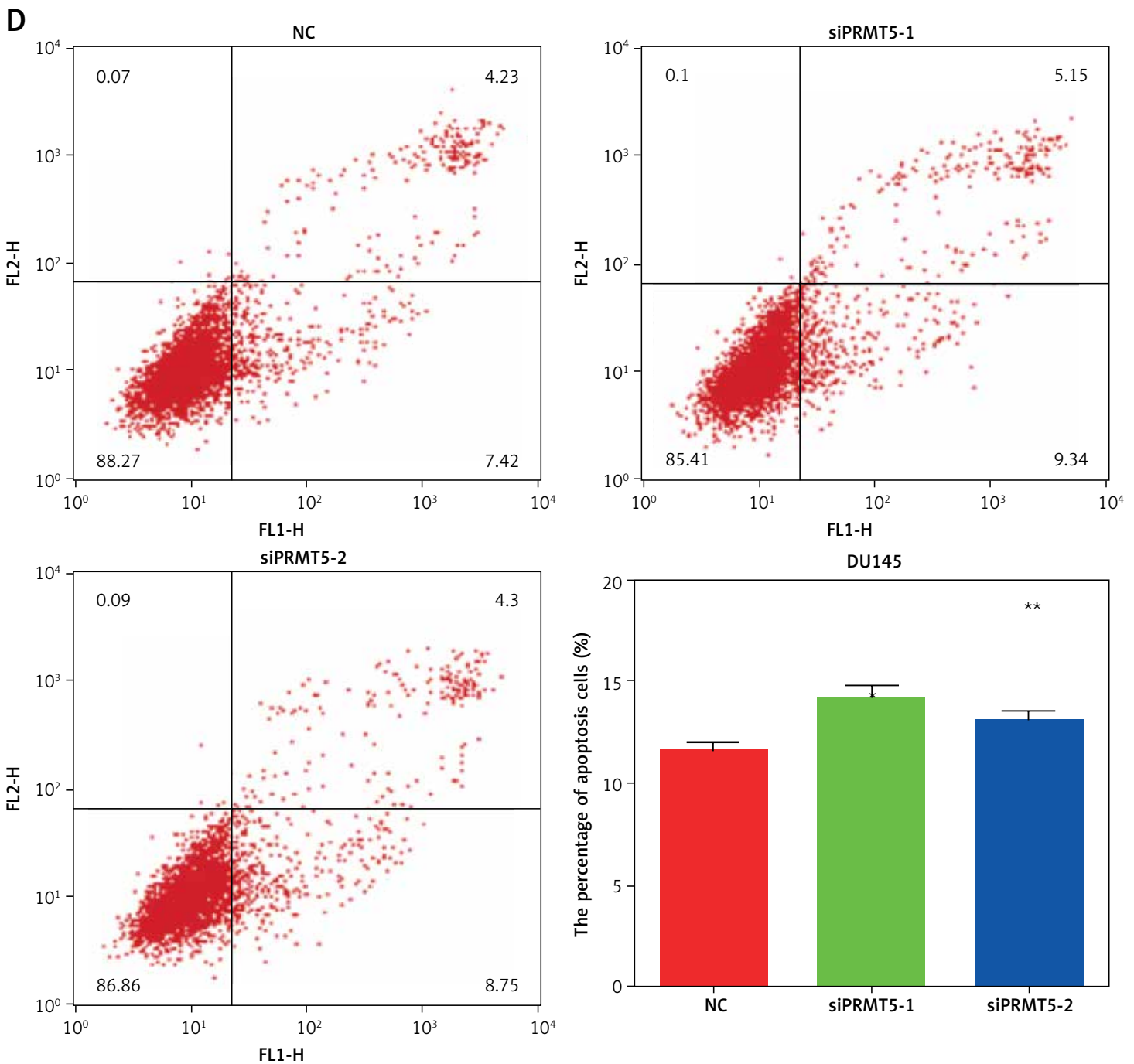

Figure 4. Cont. D - Knockdown of PRMT5 had no effect on the androgen-independent DU145 apoptosis

cell migration ability (Figures $5 \mathrm{~A}, \mathrm{~B}, \mathrm{E}, \mathrm{F}$ ). Of note, the present study found that knockdown PRMT5 significantly decreased PC-3 and DU145 cell migration ability compared to control groups in $\mathrm{PC}-3$ and DU145 cells (Figures 5 C, D, G, H).

Furthermore, the present study detected the effect of PRMT5 on cell invasion in PCa by estimating the penetration of cells through Matrigel in a transwell chamber. As illustrated in Figure 6, the present study found that knockdown of PRMT5 significantly inhibited cell invasion in DU145 and PC-3 (Figure 6 A) and PC-3 cells (Figure 6 C). The numbers of invading cells were decreased in PC-3 and DU145 cells transfected with siPRMT5, compared with the negative control group $(p<0.001$, Figures $6 \mathrm{~B}$ and D).

These results indicated that PRMT5 significantly increased invasion, and migration of androgen-independent PCa cells.

\section{Identification of the potential competing endogenous RNA networks involved in PRMT5 regulation in $\mathrm{PCa}$}

The upstream regulators of PRMT5 in PCa remained largely unknown. Long non-coding RNAs had been demonstrated as a class of key important regulators of PCa progression. The present study for the first time constructed the potential competing endogenous RNA networks involved in PRMT5 regulation in PCa. The present study first conducted a co-expression network based on the correlation analysis between the PRMT5 and differently expressed IncRNAs. The present study selected the PRMT5-IncRNA pair with the Pearson correlation coefficient $>0.4$ for further study. Then, the present study predicted potential miRNAs targeting the PRMT5-IncRNA pair using StarBase, TargetScan, and miRcode. Then, the competing endogenous RNA networks were presented using Cytoscape software. 
A
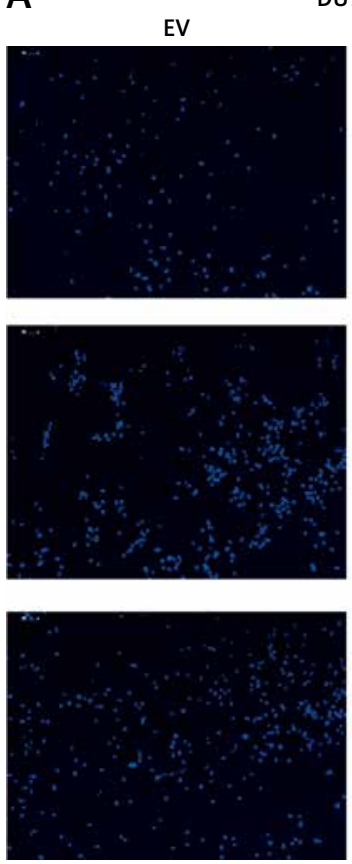

C

NC
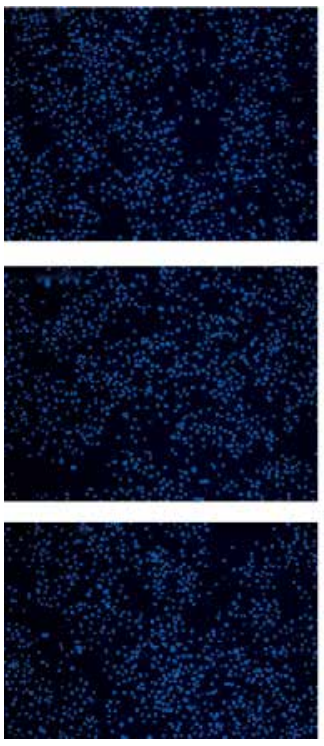

DU145
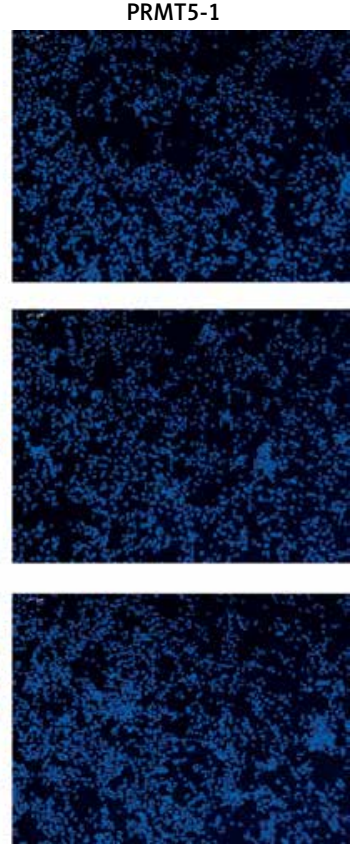

DU145 SiPRMT5-1
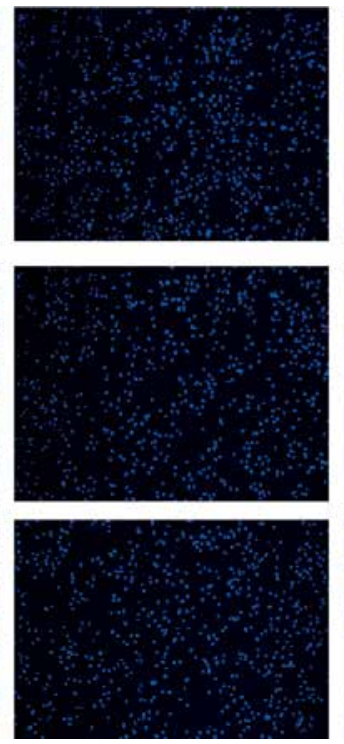

B

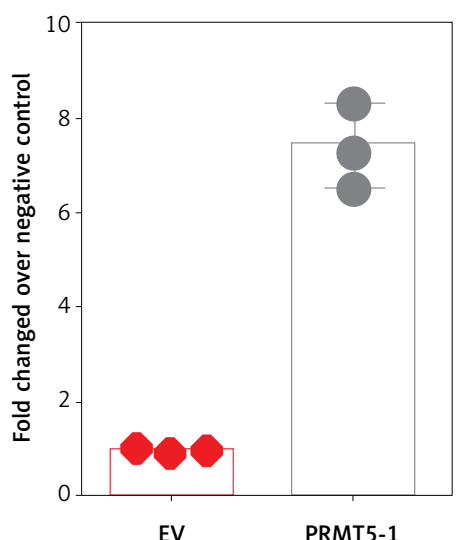

EV

D
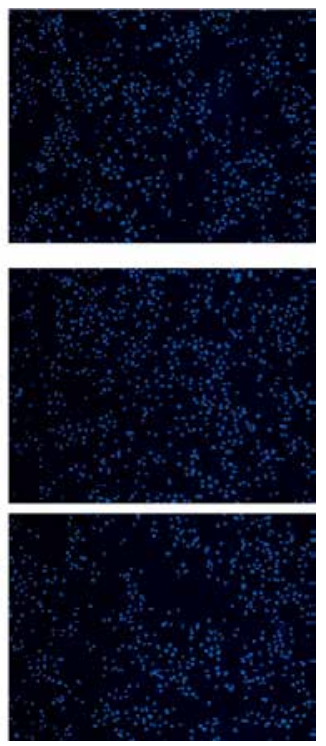

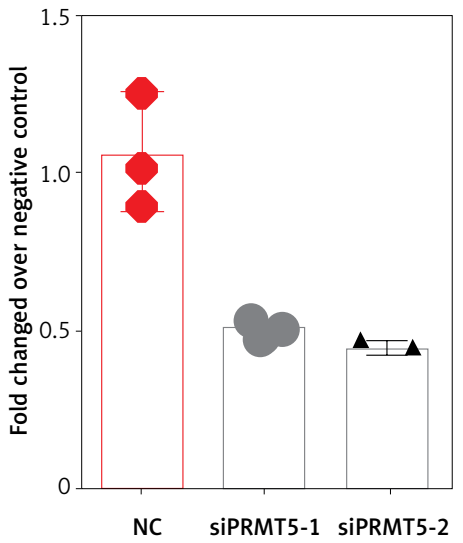

NC SIPRMT5-1 SIPRMT5-2

Figure 5. PRMT5 promotes cell migration of androgen-independent PCa. Overexpression of PRMT5 remarkably enhanced PC-3 (A, B) and DU145 (E, F) cell migration ability. Knockdown of PRMT5 significantly decreased PC-3 (C, D) and DU145 (G, H) cell migration ability compared to control groups

As shown in Figure $7 \mathrm{~A}$, a total of 17 IncRNAs (including GAS5, SNHG3, SNHG7, ZFAS1) and 26 miRNAs were included in this network. The most significantly co-expressing IncRNAS of PRMT5 in PCa included HCG18, SNHG7, SNHG8, GAS5, ZFAS1, and SNHG3 (Figure 7 B-G).

The present study focused on the ZFAS1/miR150-5p axis in PCa. ZFAS1 and miR-150-5p had been reported to be dysregulated and involved in the regulation of human cancer progression. However, their roles in PCa remained unclear.
This study for the first time showed that ZFAS1 was significantly overexpressed in PCa and correlated with shorter disease-free survival time in patients with PCa (Figures $8 \mathrm{~A}$ and B). Moreover, the present study found that the expression levels of miR-150-5p were negatively correlated with both ZFAS1 and PRMT5 expression levels in PCa (Figures $8 \mathrm{C}$ and D). These results suggested that the FAS1/miR-150-5p axis may participate in the regulation of PRMT5 expression in $\mathrm{PCa}$. 
$\mathrm{E}$
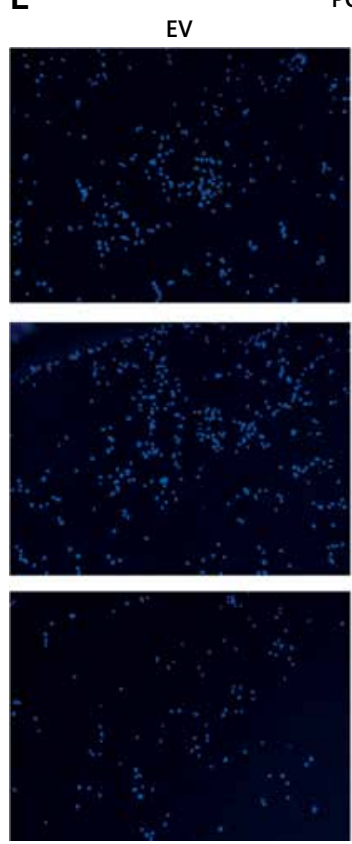

G
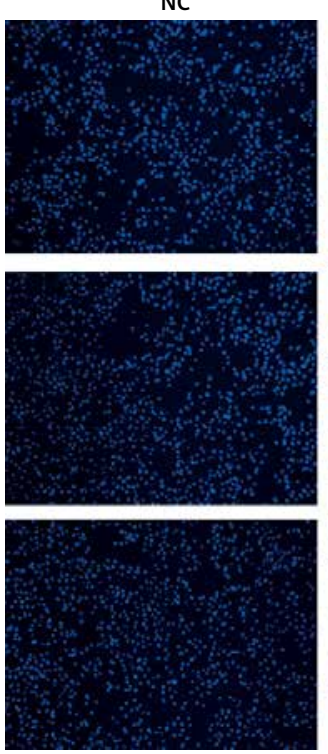

$\mathrm{PC}-3$
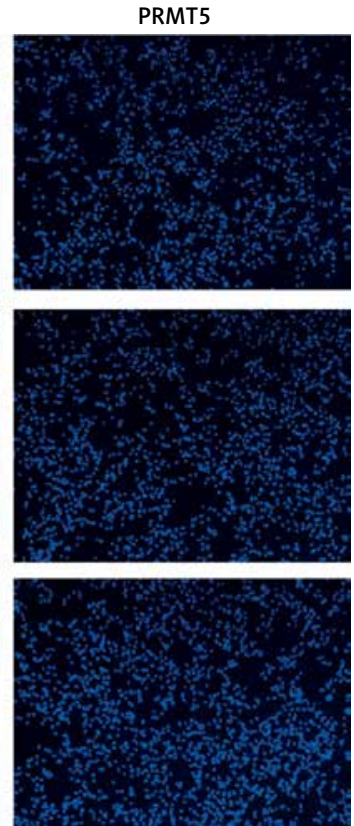

$\mathrm{F}$

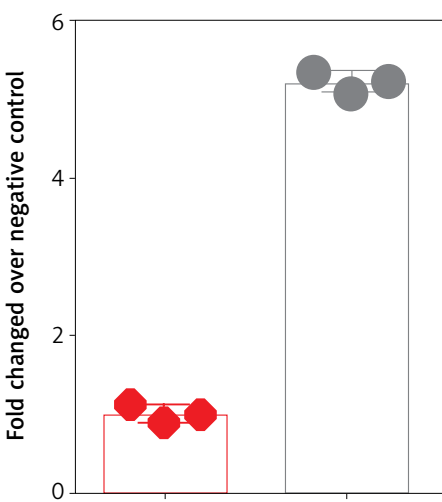

EV

PRMT5

\section{$\mathrm{H}$}
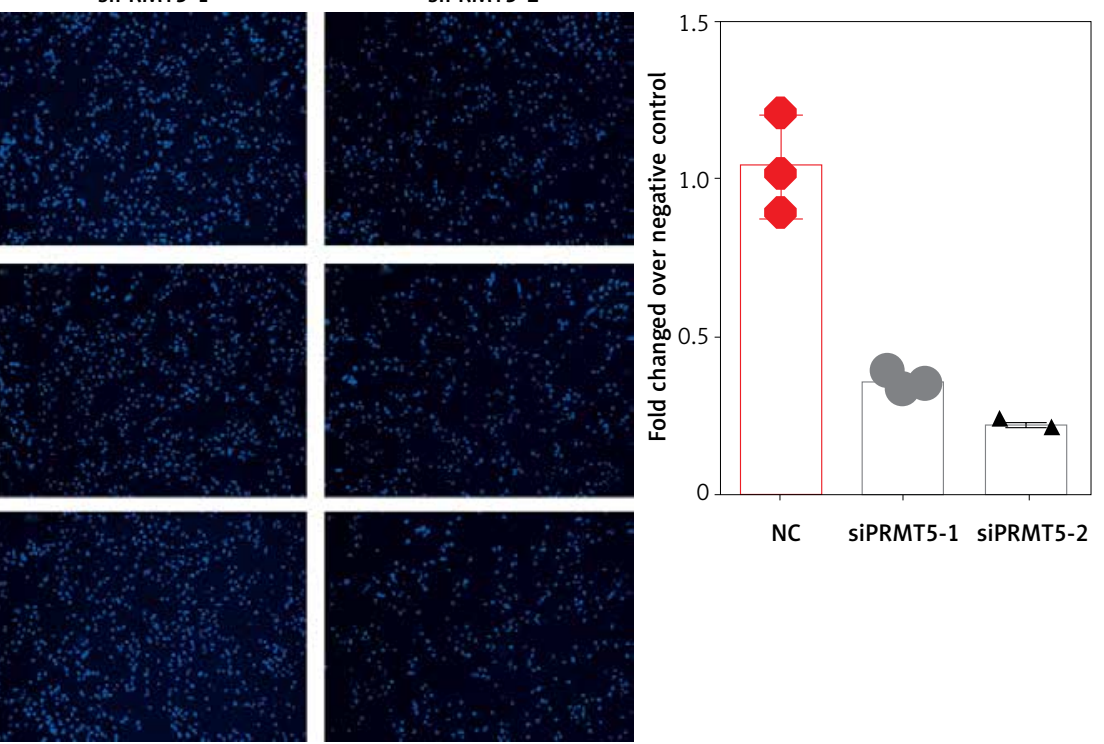

Figure 5. Cont. Overexpression of PRMT5 remarkably enhanced PC-3 (A, B) and DU145 (E, F) cell migration ability. Knockdown of PRMT5 significantly decreased PC-3 (C, D) and DU145 (G, H) cell migration ability compared to control groups

ZFAS1 acted as a ceRNA to up-regulate PRMT5 expression though miR-150-5p

Then, the present study detected the expression levels of ZFAS1 and PRMT5 after overexpressing miR-150-5p in PC-3 and DU145 cells. As presented in Figures $8 \mathrm{E}$ and $\mathrm{G}$, our results showed that the RNA levels of ZFAS1 and PRMT5 were significantly reduced after overexpressing miR-150-5p in PC-3 and DU145 cells using RTPCR assay. Western blot assay also showed that the protein levels of PRMT5 were significantly decreased in PC-3 and DU145 cells transfected with miR-150-5p compared to normal samples (Figure $8 \mathrm{H}$ ).

Furthermore, the direct interaction between miR-150-5p and PRMT5 or ZFAS1 was detected using luciferase assays. The luciferase reporter assay revealed that luciferase activity was significantly repressed in the constructs of the ZFAS1 (Figure $8 \mathrm{~F}$ ) and PRMT5 -3'UTR (Figure 8 I) when co-transfected with the corresponding miRNAs compared 
A
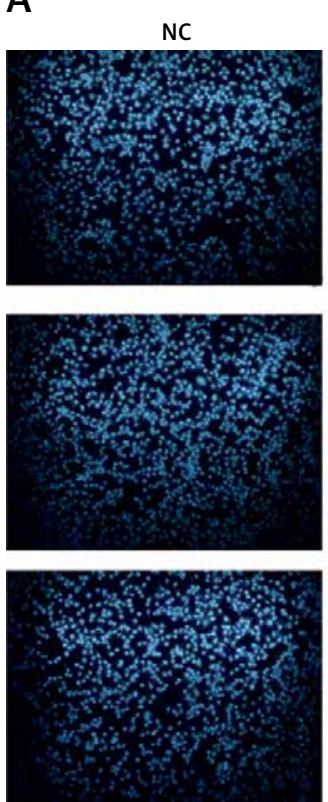

C
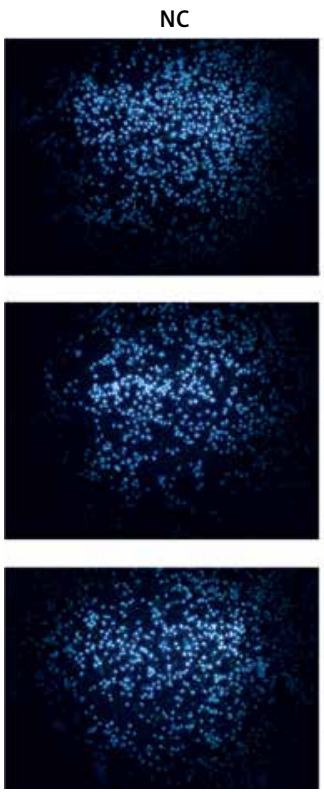

DU145
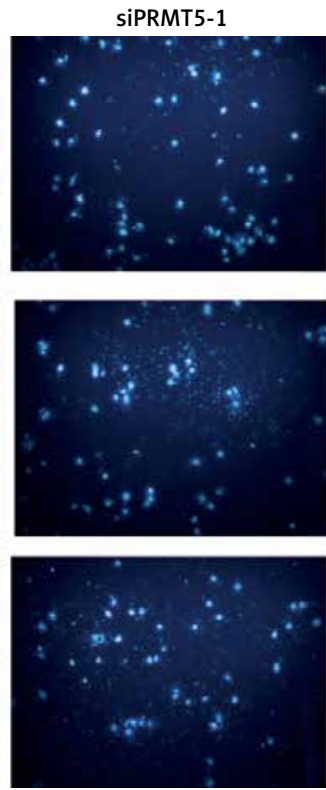

PC-3

SIPRMT5-1
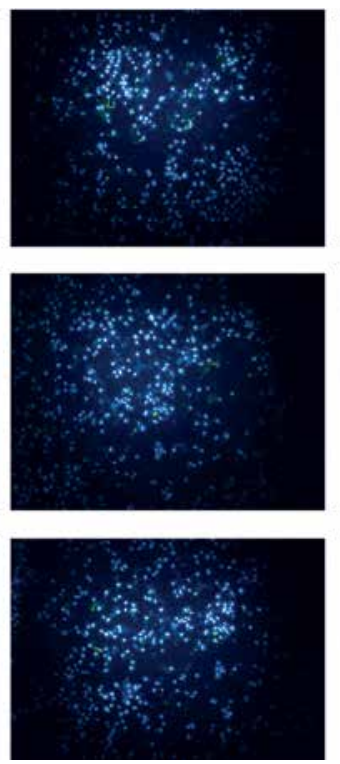

B
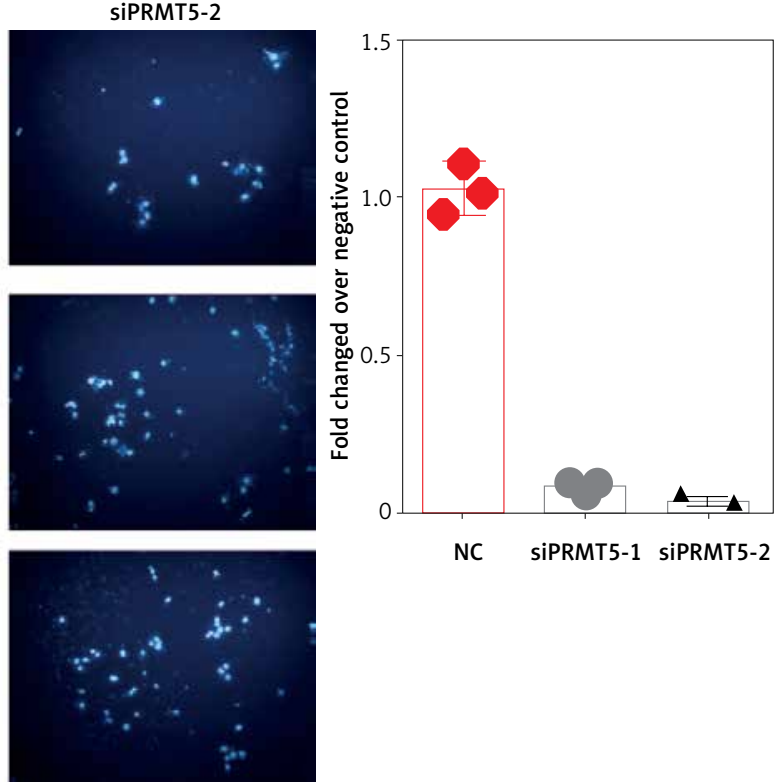

D
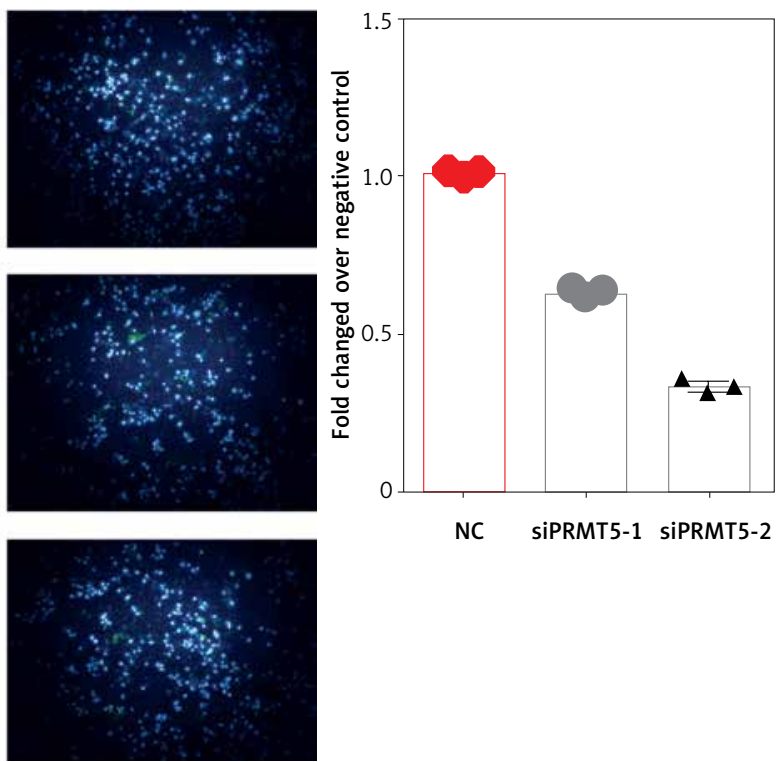

SiPRMT5-2
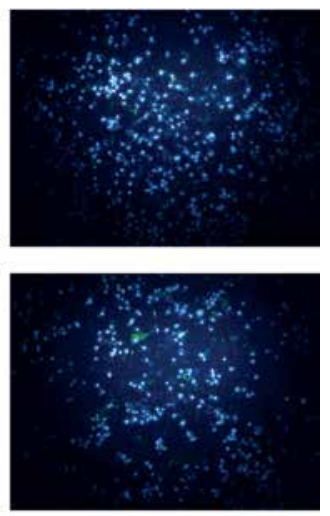

Figure 6. PRMT5 promotes cell invasion of androgen-independent PCa. Knockdown of PRMT5 significantly inhibited cell invasion in DU145 (A, B) and PC-3 cells (C, D)

with NC, whereas the mutated 3'UTR did not show a significant response to miR-150-5p.

\section{ZFAS1 promoted cell migration and} invasion of PCa though PRMT5

As presented in Figures $9 \mathrm{~A}$ and $\mathrm{B}$, the present study found that knockdown of ZFAS1 significantly inhibited migration of PC-3 5 cells by $75 \%$ compared with the NC group, respectively. Moreover, knockdown of ZFAS1 significantly inhibited invasion of PC-3 cells by $47 \%$ compared with the NC group (Figures $9 \mathrm{C}$ and D), respectively.
To test whether ZFAS1 promoted cell migration and invasion of PCa though PRMT5, the present study conducted rescue experiments. PC-3 cells co-transfected with siZFAS1 and PRMT5 significantly enhanced the PC-3 cell migration ability compared to the cells only transfected with siZFAS1 (Figures 9 A-D).

\section{Discussion}

PRMT5 was regarded as an oncogene in human cancers by regulating multiple cancer related regulators, such as TP53 and PDCD4 [26]. For example, 
A

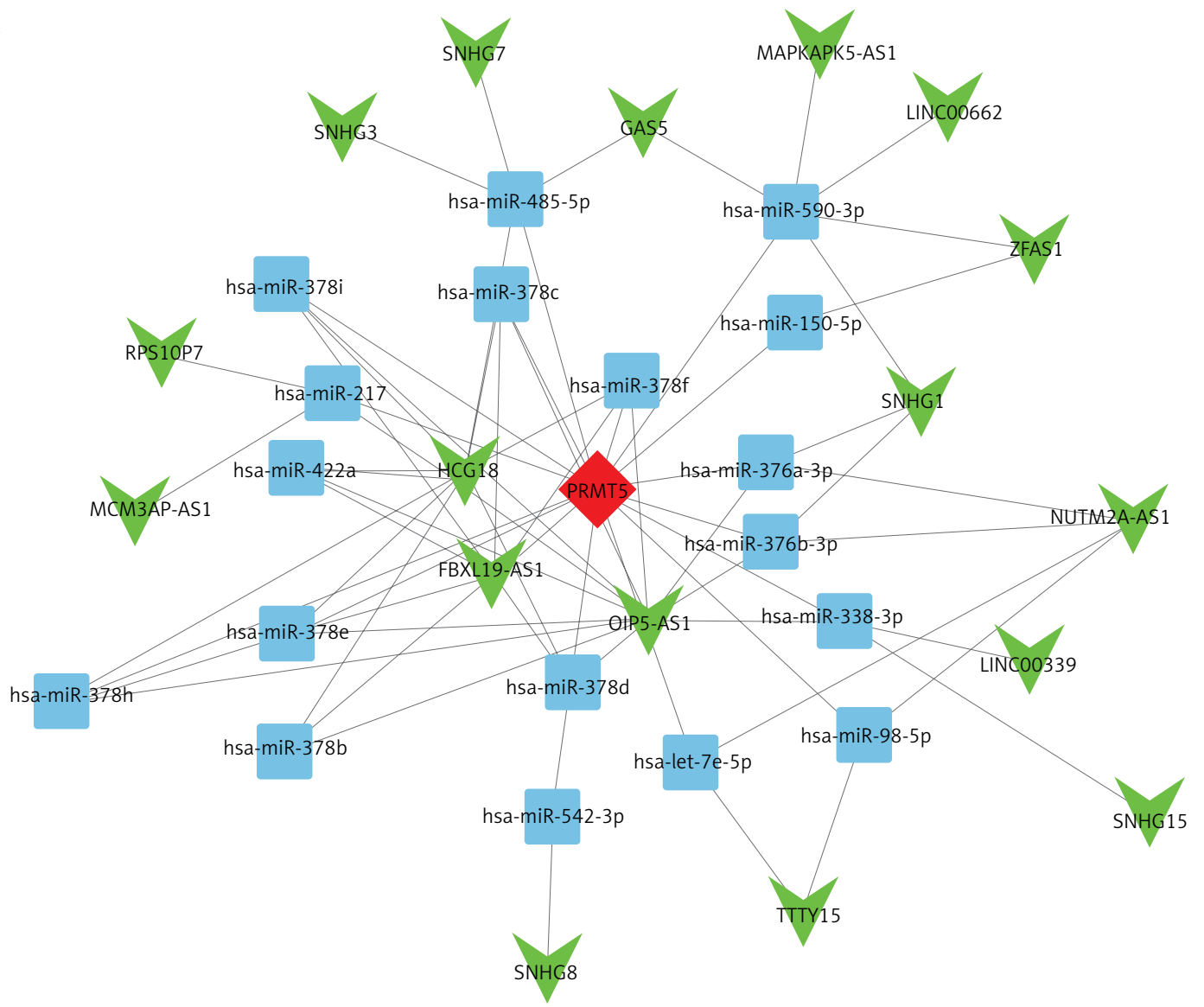

B
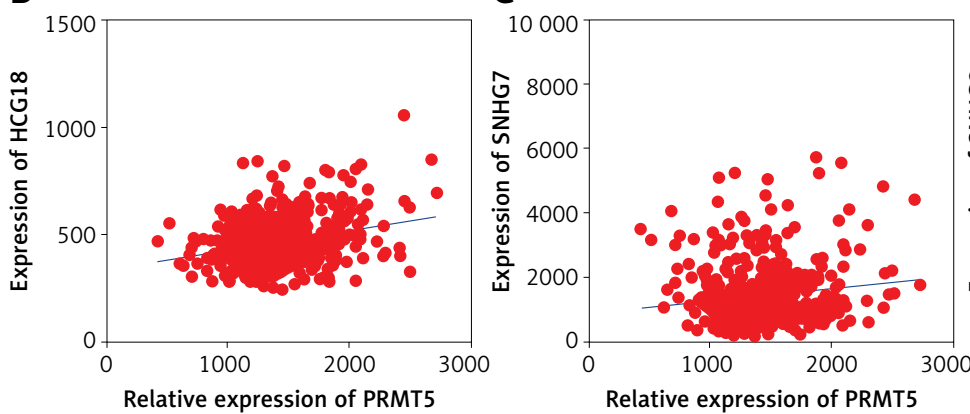

$\mathbf{F}$

E

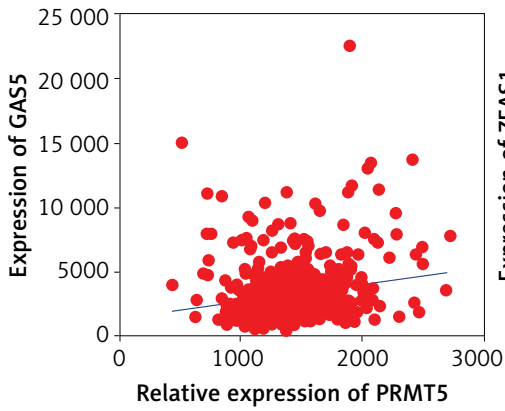

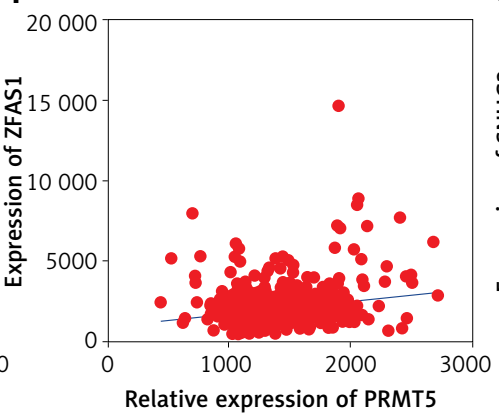

D

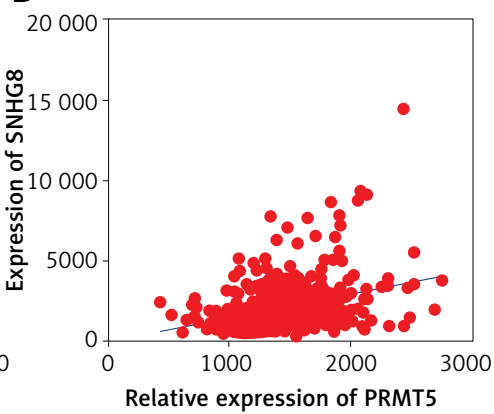

G

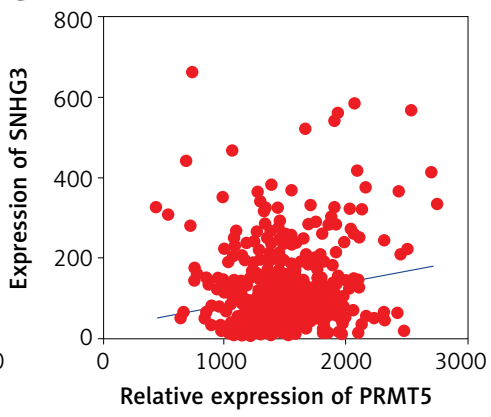

Figure 7. The potential competing endogenous RNA networks involved in PRMT5 regulation in PCa. A - A total of 17 IncRNAs (including GAS5, SNHG3, SNHG7, ZFAS1) and 26 miRNAs were included in this network. B-G - The most significantly co-expressing IncRNAS of PRMT5 in PCa included HCG18, SNHG7, SNHG8, GAS5, ZFAS1, and SNHG3 
A

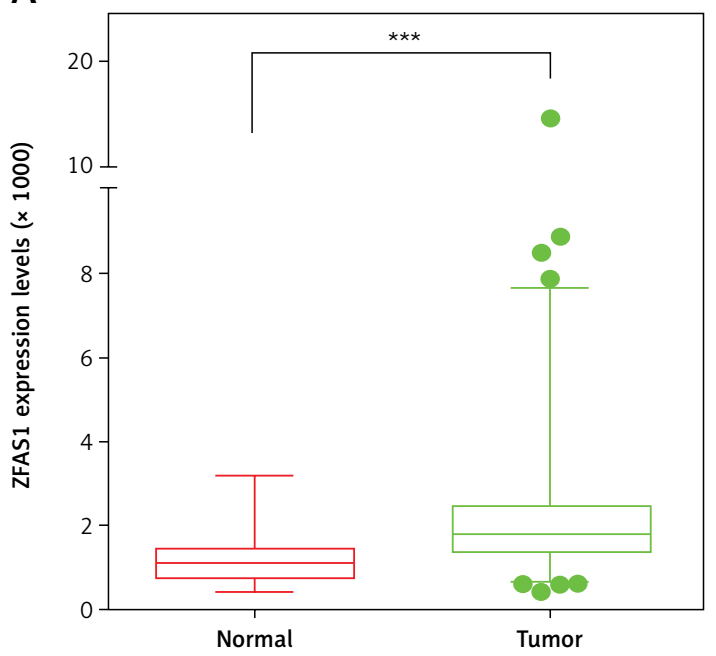

C

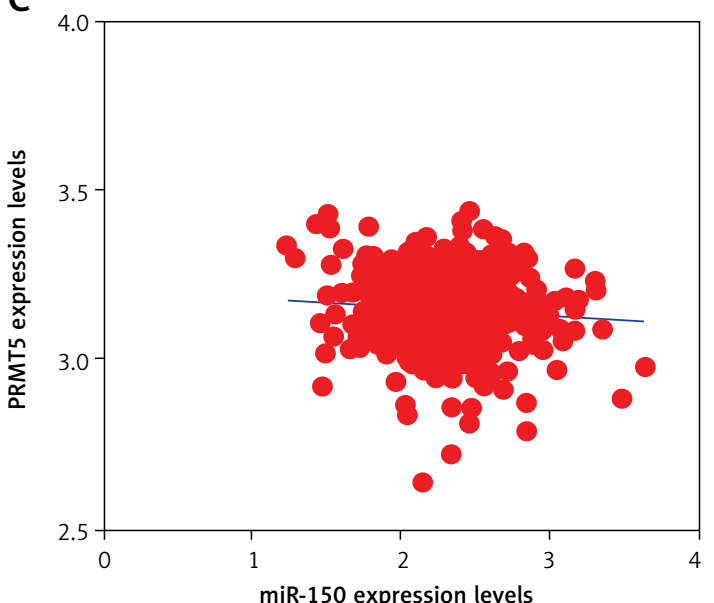

$\mathrm{E}$

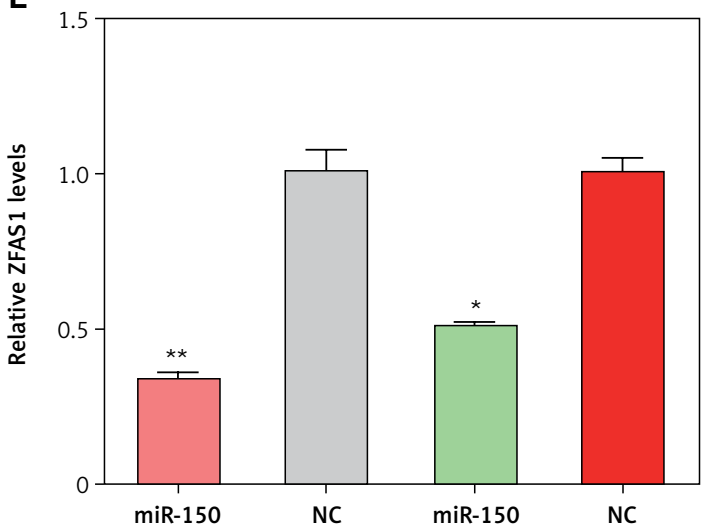

B

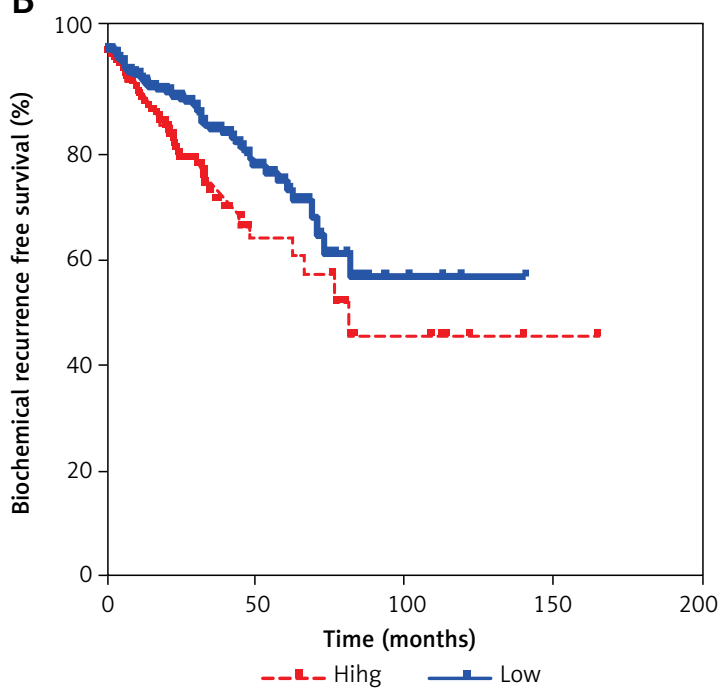

D

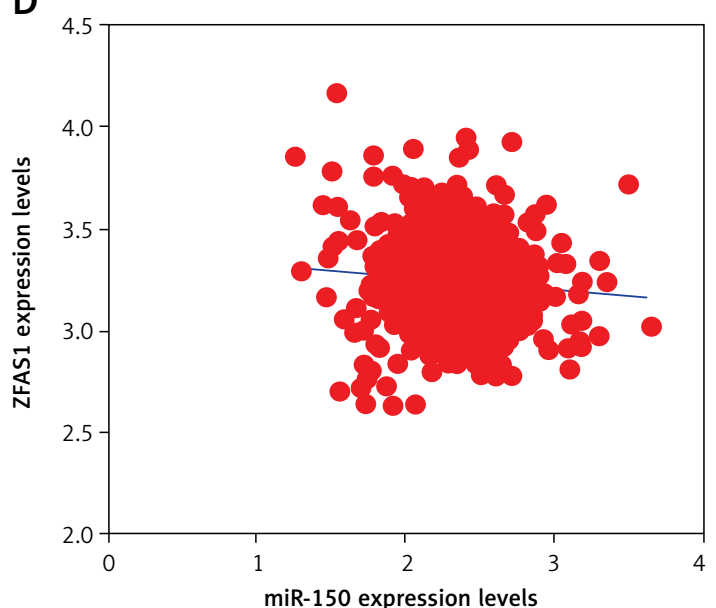

$\mathrm{F}$

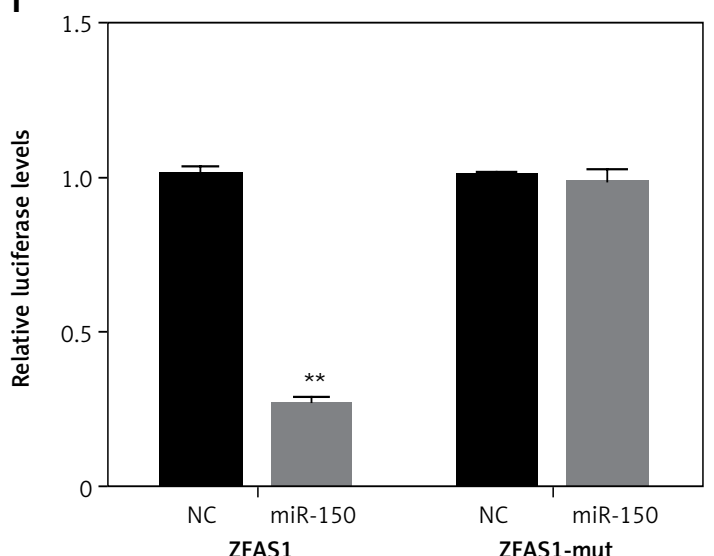

Figure 8. ZFAS1 acted as a ceRNA to up-regulate PRMT5 expression though miR-150-5p. A - ZFAS1 was significantly overexpressed in PCa. B - ZFAS1 was correlated with shorter disease-free survival time in patients with PCa. C - The expression level of miR-150-5p was negatively correlated with ZFAS1 expression levels in PCa. D - The expression level of miR-150-5p was negatively correlated with PRMT5 expression levels in PCa. E - Overexpression of miR-150-5p significantly reduced the RNA levels of ZFAS1 in PC-3 and DU145 cells. F - Overexpression of miR150-5p significantly decreased the luciferase activity of ZFAS1 
G

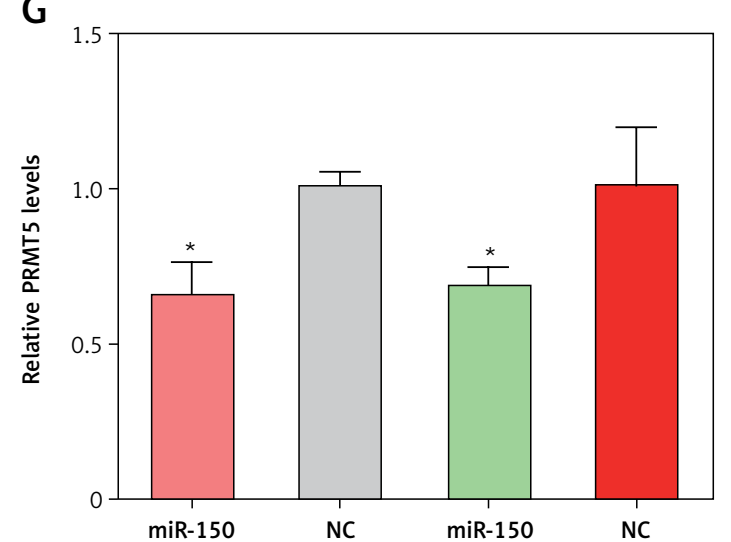

I

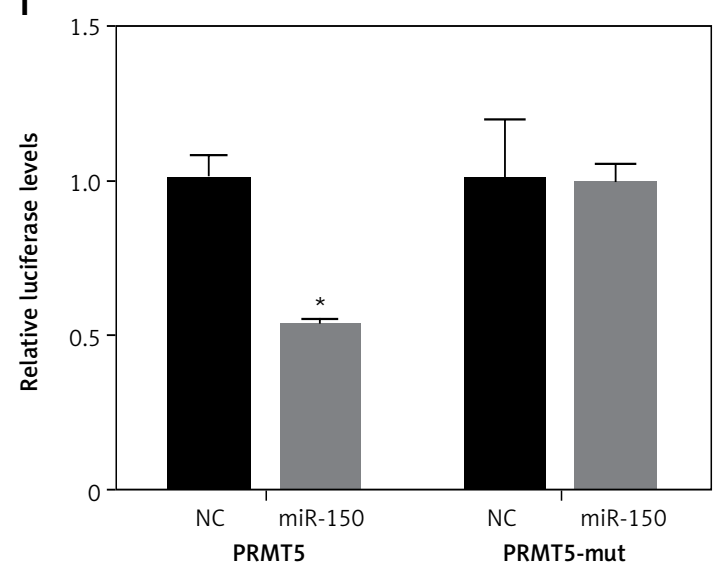

the methylation of p53 by PRMT5 inhibits the expression of pro-apoptotic and anti-proliferative genes, thereby maintaining tumor cell self-renewal and proliferation. The methylation of E2F1 mediated by PRMT5 suppressed its activity to inhibit cancer proliferation [27]. PRMT5 has been observed to be overexpressed in various human cancers, such as melanoma, lung cancer, ovarian cancer, breast cancer and prostate cancer [21]. Of note, recent studies indicated that PRMT5 plays crucial roles in PCa tumorigenesis and development though affecting AR. AR is the most important regulator in PCa progression. Previous studies showed that PRMT5 promotes PCa cell growth by epigenetic activation of AR transcription. Inhibition of PRMT5 down-regulates AR expression and inhibits the growth of multiple AR-positive PCa cells [22, 24]. However, the effects of PRMT5 on $\mathrm{PCa}$ metastasis remained unclear. The present study conducted loss/gain of function assay to evaluate the roles of PRMT5 in PCa. Our results showed that PRMT5 significantly promoted androgen-dependent PCa proliferation and cell cycle progression and suppressed cell apoptosis. However, PRMT5 did not affect androgen-independent PCa proliferation. These results were consistent with previous reports and AR may play a key role in the regulation of PRMT5 functions in andro-
$\mathrm{H}$

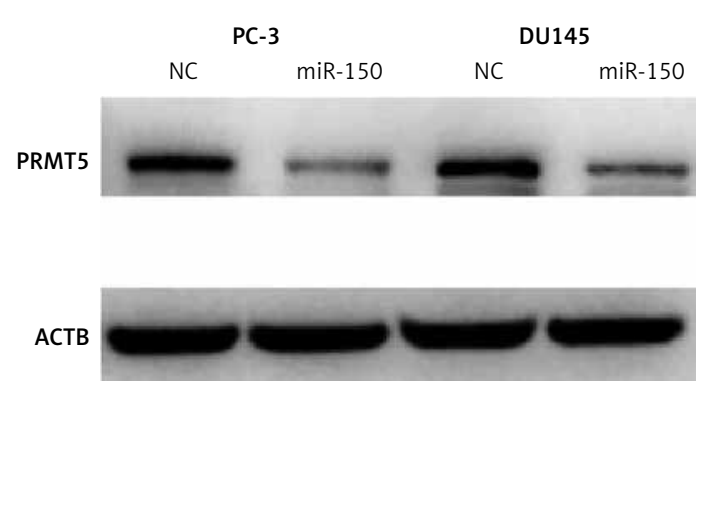

Figure 8. Cont. G - Overexpression of miR-150-5p significantly reduced the RNA levels of PRMT5 in PC-3 and DU145 cells. $\mathrm{H}-$ Overexpression of miR150-5p significantly reduced the protein levels of PRMT5 in PC-3 and DU145 cells. I - Overexpression of miR-150-5p significantly decreased the luciferase activity of PRMT5

gen-dependent PCa. Very interestingly, this study showed that PRMT5 could significantly induce androgen-independent PCa metastasis. Knockdown of PRMT5 suppressed, whereas overexpression of PRMT5 induced, cell migration and invasion in androgen-independent DU145 and PC-3 cells.

The up-stream regulators of PRMT5 remained largely unclear. A previous study showed that nuclear factor $Y$ (NF-Y) transcriptional activation of PRMT5 is required for cell growth and is regulated by PKC /c-Fos signaling in prostate cancer cells [21]. Several miRNAs, such as miR-92b and miR-96, were also reported to be involved in the post-transcriptional regulation of PRMT5 in human cancers [28]. The present study for the first time showed that long non-coding RNAs were also involved in promoting PRMT5 expression in PCa though ceRNA mechanisms. LncRNAs are a novel class of RNA transcripts longer than 200 nucleotides. LncRNAs were found to be dysregulated in multiple cancers and regulated cancer related processes though binding to miRNAs, protein and DNAs. For example, IncRNA HULC promote PCa progression [29], IncRNA PVT1 promote multiple myeloma progression by inhibiting miR-203a expression [30], and IncRNA MIAT promoted epithelial ovarian cell proliferation and inhibited cell apoptosis by inhibiting miR-330-5p expression [31]. The most well- 
A
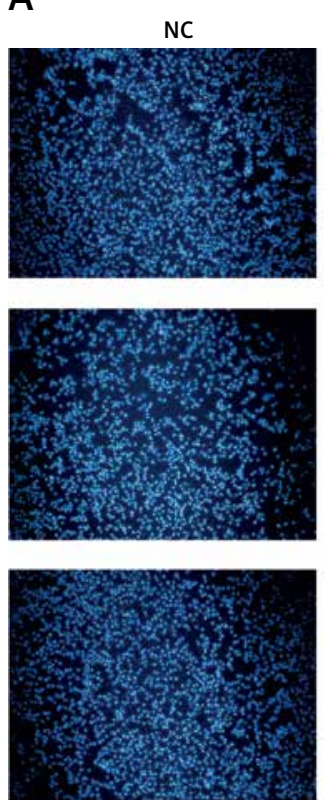

C
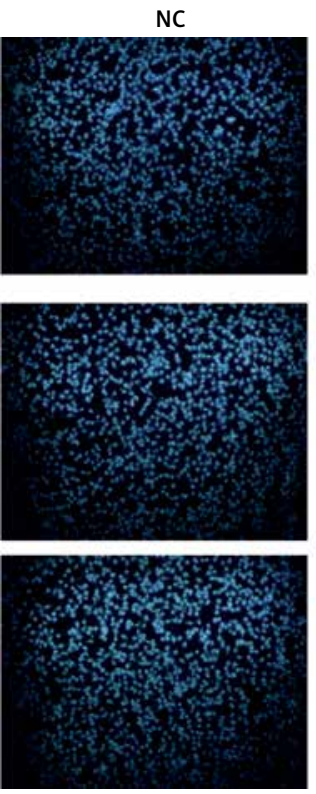

Migration
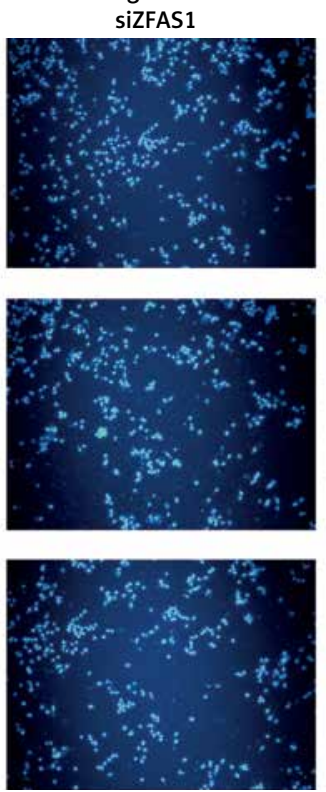

Invasion

siZFAS1
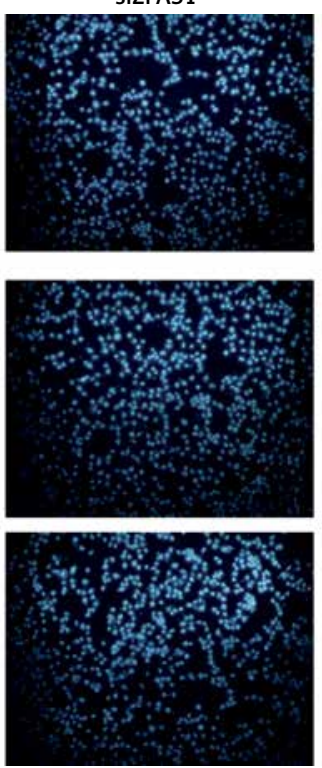

B
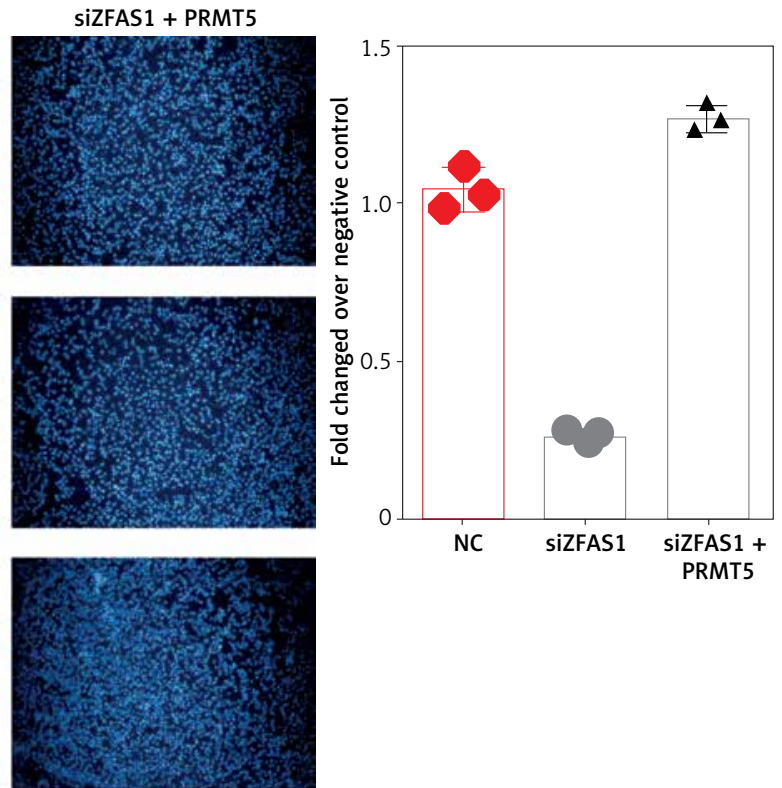

D

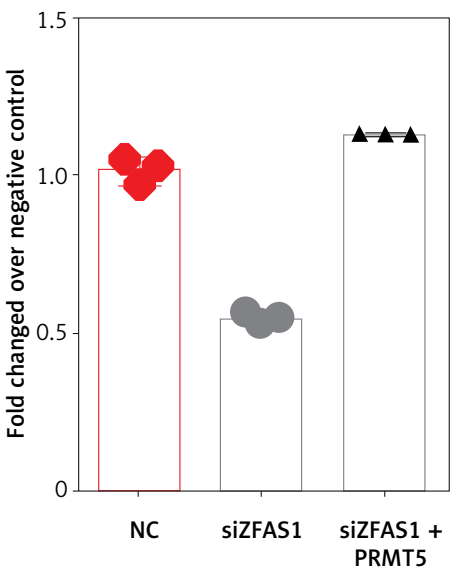

Figure 9. ZFAS1 promoted cell migration and invasion of PCa though PRMT5. A, B - Knockdown of ZFAS1 significantly inhibited migration of PC-3 cells compared with NC group; overexpression of PRMT5 could rescue the migration. C, D - Knockdown of ZFAS1 significantly inhibited invasion of PC-3 cells compared with NC group; overexpression of PRMT5 could rescue the invasion

known mechanism of IncRNAs regulating targets is the ceRNA mechanism [32]. Also, ceRNAs show importance in regulating cancer progression [33]. For example, SNHG20 promotes PCa cell proliferation and migration via acting as a ceRNA to upregulate DDX17 [34]. The present study conducted bioinformatics analysis and constructed a ceRNA network involved in regulating PRMT5 expression in PCa. The further validation showed that ZFAS1 could up-regulate PRMT5 expression though sponging miR-150-5p.
LncRNA ZFAS1 had been reported to be up-regulated in several types of cancers, including hepatocellular carcinoma, colorectal cancer, gastric cancer, and glioma [35]. For example, ZFAS1 promotes tumorigenesis through regulation of miR150-5p/RAB9A in melanoma [36]. Knockdown of ZFAS1 suppresses the progression of acute myeloid leukemia by regulating microRNA-150/ Sp1 and microRNA-150/Myb pathways [37]. SP1-induced ZFAS1 contributes to colorectal cancer progression via the miR-150-5p/VEGFA axis 
[38]. In ovarian cancer, ZFAS1 was reported to promote ovarian cancer cell malignancy by interacting with miR-150-5p [39]. These reports were consistent with our finding that ZFAS1 could interact with miR-150-5p in PCa. Of note, this study for the first time demonstrated that ZFAS1 acted as a metastasis promoter in PCa. Knockdown of ZFAS1 could significantly reduce PCa migration and invasion. Moreover, our study showed that ZFAS1 promoted cell migration and invasion of PCa though PRMT5. Interestingly, our study analyzed the expression levels of ZFAS1, miR-150$5 p$ and PRMT5 in PCa using a TCGA dataset. Our results showed that ZFAS1 and PRMT5 were overexpressed and miR-150-5p was down-regulated in PCa samples. Higher expression of ZFAS1 and PRMT5 was correlated with shorter disease free survival time in $\mathrm{PC}$ a patients. Moreover, the present study found that the expression levels of miR-150-5p were negatively correlated with both ZFAS1 and PRMT5 expression levels in PCa. These results suggested that the ZFAS1/miR150-5p axis may participate in the regulation of PRMT5 expression in PCa.

In conclusion, the present study showed that PRMT5 promoted androgen-dependent PCa proliferation and androgen-independent PCa migration and invasion. PRMT5 knockdown decreased and PRMT5 overexpression enhanced DU145 and PC3 migration and invasion. Moreover, our results showed that the ZFAS1/miR-150-5p axis regulated PRMT5 expression in PCa cells. Furthermore, the present study showed ZFAS1 and PRMT5 were overexpressed and miR-150-5p was down-regulated in PCa samples. Higher expression of ZFAS1 and PRMT5 was correlated with shorter disease free survival time in $\mathrm{PCa}$ patients. These results showed that PRMT5 may be a therapeutic target for PCa.

\section{Acknowledgement}

Hao Shen and Lei Wang have contributed equally to this work.

This study was supported by the National Natural Science Foundation of China (No. 81972408), Application and Basic Research Project of Wuhan City (No. 2018060401011321), Wuhan Morning Light Plan of Youth Science and Technology (2017050304010281), Hubei Province Health and Family Planning Scientific Research Project (No. WJ2017M025 No. WJ2017Z005 and No. WJ2018H209), Natural Science Foundation of Hubei Province (No. 2016CFB114, 2017CFB181) and Research Project of Wuhan University (No. 2042017kf0097).

\section{Conflict of interest}

The authors declare no conflict of interest.

\section{References}

1. Hamamoto R, Saloura V, Nakamura Y. Critical roles of non-histone protein lysine methylation in human tumorigenesis. Nat Rev Cancer 2015; 15: 110-24.

2. Colon-Bolea P, Crespo P. Lysine methylation in cancer: SMYD3-MAP3K2 teaches us new lessons in the Ras-ERK pathway. Bioessays 2014; 36: 1162-9.

3. Hamamoto R, Toyokawa G, Nakakido M, Ueda K, Nakamura Y. SMYD2-dependent HSP90 methylation promotes cancer cell proliferation by regulating the chaperone complex formation. Cancer Lett 2014; 351: 126-33.

4. Viktorsson K, Lewensohn R, Zhivotovsky B. Systems biology approaches to develop innovative strategies for lung cancer therapy. Cell Death Dis 2014; 5: e1260.

5. Banyra O, Tarchynets M, Shulyak A. Renal cell carcinoma: how to hit the targets? Centr European J Urol 2014; 66: 394-404.

6. Guccione E, Richard S. The regulation, functions and clinical relevance of arginine methylation. Nat Rev Mol Cell Biol 2019; 20: 642-57.

7. Yang Y, Bedford MT. Protein arginine methyltransferases and cancer. Nat Rev Cancer 2013; 13: 37-50.

8. Bray F, Ferlay J, Soerjomataram I, Siegel RL, Torre LA, Jemal A. Global cancer statistics 2018: GLOBOCAN estimates of incidence and mortality worldwide for 36 cancers in 185 countries. CA Cancer J Clin 2018; 68: 394-424.

9. Lima AR, Bastos ML, Carvalho M, Guedes DPP. Biomarker discovery in human prostate cancer: an update in metabolomics studies. Transl Oncol 2016; 9: 357-70.

10. Vaz CV, Marques R, Alves MG, et al. Androgens enhance the glycolytic metabolism and lactate export in prostate cancer cells by modulating the expression of GLUT1, GLUT3, PFK, LDH and MCT4 genes. J Cancer Res Clin Oncol 2016; 142: 5-16.

11. Hjorth-Jensen K, Maya-Mendoza A, Dalgaard N, et al. SPOP promotes transcriptional expression of DNA repair and replication factors to prevent replication stress and genomic instability. Nucleic Acids Res 2018; 46: 9484-95. Erratum: 9891.

12. Yan M, Qi H, Li J, et al. Identification of SPOP related metabolic pathways in prostate cancer. Oncotarget 2017; 8: 103032-46.

13. Barbieri CE, Demichelis F, Rubin MA. Molecular genetics of prostate cancer: emerging appreciation of genetic complexity. Histopathology 2012; 60: 187-98.

14. Cha B, Jho EH. Protein arginine methyltransferases (PRMTs) as therapeutic targets. Expert Opin Ther Targets 2012; 16: 651-64.

15. Baldwin RM, Morettin A, Cote J. Role of PRMTs in cancer: Could minor isoforms be leaving a mark? World J Biol Chem 2014; 5: 115-29.

16. Stein C, Riedl S, Ruthnick D, Notzold RR, Bauer UM. The arginine methyltransferase PRMT6 regulates cell proliferation and senescence through transcriptional repression of tumor suppressor genes. Nucleic Acids Res 2012; 40: 9522-33.

17. Harada N, Takagi T, Nakano Y, Yamaji R, Inui H. Protein arginine methyltransferase 10 is required for andro- 
gen-dependent proliferation of LNCaP prostate cancer cells. Biosci Biotechnol Biochem 2015; 79: 1430-7.

18. Rho J, Choi S, Seong YR, Cho WK, Kim SH, Im DS. PRMT5, which forms distinct homo-oligomers, is a member of the protein-arginine methyltransferase family. J Biol Chem 2001; 276: 11393-401.

19. Timm DE, Bowman V, Madsen R, Rauch C. Cryo-electron microscopy structure of a human PRMT5: MEP50 complex. PLoS One 2018; 13: e193205.

20. Scoumanne A, Zhang J, Chen X. PRMT5 is required for cell-cycle progression and p53 tumor suppressor func tion. Nucleic Acids Res 2009; 37: 4965-76.

21. Zhang HT, Zhang D, Zha ZG, Hu CD. Transcriptional activation of PRMT5 by NF-Y is required for cell growth and negatively regulated by the $\mathrm{PKC} / \mathrm{c}-$ Fos signaling in prostate cancer cells. Biochim Biophys Acta 2014; 1839: 1330-40.

22. Deng X, Shao G, Zhang HT, et al. Protein arginine methyltransferase 5 functions as an epigenetic activator of the androgen receptor to promote prostate cancer cell growth. Oncogene 2017; 36: 1223-31.

23. Hu C, Deng X, Beketova E, Owens J, Malola J. Cotargeting of Androgen Synthesis and Androgen Receptor Expression as a Novel Treatment for Castration Resistant Prostate Cancer. Purdue University West Lafayette, United States, 2017.

24. Mounir Z, Korn JM, Westerling T, et al. ERG signaling in prostate cancer is driven through PRMT5-dependent methylation of the androgen receptor. Elife 2016; 5: e13964.

25. Livak KJ, Schmittgen TD. Analysis of relative gene expression data using real-time quantitative PCR and the 2(-Delta Delta C(T)) Method. Methods 2001; 25: 402-8.

26. Richters $A$. Targeting protein arginine methyltransferase 5 in disease. Future Med Chem 2017; 9: 2081-98.

27. Putzer BM, Engelmann D. E2F1 apoptosis counterattacked: evil strikes back. Trends Mol Med 2013; 19: 89-98

28. Pal S, Baiocchi RA, Byrd JC, Grever MR, Jacob ST, Sif S. Low levels of miR-92b/96 induce PRMT5 translation and $\mathrm{H} 3 \mathrm{R} 8 / \mathrm{H} 4 \mathrm{R} 3$ methylation in mantle cell lymphoma. EMBO J 2007; 26: 3558-69.

29. Zheng P, Li H, Xu P, et al. High IncRNA HULC expression is associated with poor prognosis and promotes tumor progression by regulating epithelial-mesenchymal transition in prostate cancer. Arch Med Sci 2018; 14: 679-86.

30. Yang M, Zhang L, Wang X, Zhou Y, Wu S. Down-regulation of miR-203a by IncRNA PVT1 in multiple myeloma promotes cell proliferation. Arch Med Sci 2018; 14: 1333-9.

31. Shao S, Tian J, Zhang H, Wang S. LncRNA myocardial infarction-associated transcript promotes cell proliferation and inhibits cell apoptosis by targeting miR-330-5p in epithelial ovarian cancer cells. Arch Med Sci 2018; 14: 1263-70.

32. Tay Y, Rinn J, Pandolfi PP. The multilayered complexity of ceRNA crosstalk and competition. Nature 2014; 505: 344-52.

33. Qi X, Zhang DH, Wu N, Xiao JH, Wang X, Ma W. ceRNA in cancer: possible functions and clinical implications. J Med Genet 2015; 52: 710-8.

34. Wu X, Yan W, Ji Z, Zheng G, Liu G. Long noncoding RNA SNHG2O promotes prostate cancer progression via upregulating DDX17. Arch Med Sci 2019; https://doi. org/10.5114/aoms.2019.85653.
35. Dong D, Mu Z, Zhao C, Sun M. ZFAS1: a novel tumor-related long non-coding RNA. Cancer Cell Int 2018; 18: 125.

36. Liang L, Zhang Z, Qin X, et al. Long noncoding RNA ZFAS1 promotes tumorigenesis through regulation of miR-150-5p/RAB9A in melanoma. Melanoma Res 2019; 29: $569-81$

37. Gan S, Ma P, Ma J, et al. Knockdown of ZFAS1 suppresses the progression of acute myeloid leukemia by regulating microRNA-150/Sp1 and microRNA-150/Myb pathways. Eur J Pharmacol 2019; 844: 38-48.

38. Chen X, Zeng K, Xu M, et al. SP1-induced IncRNA-ZFAS1 contributes to colorectal cancer progression via the miR-150-5p/VEGFA axis. Cell Death Dis 2018; 9: 982.

39. Xia B, Hou Y, Chen H, et al. Long non-coding RNA ZFAS1 interacts with miR-150-5p to regulate Sp1 expression and ovarian cancer cell malignancy. Oncotarget 2017; 8: 19534-46. 